\title{
Dynamic Probabilistic CCA for Analysis of Affective Behavior and Fusion of Continuous Annotations
}

\author{
Mihalis A. Nicolaou, Student Member, IEEE, Vladimir Pavlovic, Member, IEEE, and \\ Maja Pantic, Fellow, IEEE
}

\begin{abstract}
Fusing multiple continuous expert annotations is a crucial problem in machine learning and computer vision, particularly when dealing with uncertain and subjective tasks related to affective behavior. Inspired by the concept of inferring shared and individual latent spaces in Probabilistic Canonical Correlation Analysis (PCCA), we propose a novel, generative model that discovers temporal dependencies on the shared/individual spaces (Dynamic Probabilistic CCA, DPCCA). In order to accommodate for temporal lags, which are prominent amongst continuous annotations, we further introduce a latent warping process, leading to the DPCCA with Time Warpings (DPCTW) model. Finally, we propose two supervised variants of DPCCA/DPCTW which incorporate inputs (i.e. visual or audio features), both in a generative (SG-DPCCA) and discriminative manner (SD-DPCCA). We show that the resulting family of models (i) can be used as a unifying framework for solving the problems of temporal alignment and fusion of multiple annotations in time, (ii) can automatically rank and filter annotations based on latent posteriors or other model statistics, and (iii) that by incorporating dynamics, modeling annotation-specific biases, noise estimation, time warping and supervision, DPCTW outperforms state-of-the-art methods for both the aggregation of multiple, yet imperfect expert annotations as well as the alignment of affective behavior.
\end{abstract}

Index Terms-Fusion of continuous annotations, component analysis, temporal alignment, dimensional emotion, affect analysis

\section{INTRODUCTION}

$\mathrm{M}$ OST supervised learning tasks in computer vision and machine learning assume the existence of a reliable, objective label that corresponds to a given training instance. Nevertheless, especially in problems related to human behavior, the annotation process (typically performed by multiple experts to reduce individual bias) can lead to inaccurate, ambiguous and subjective labels which in turn are used to train ill-generalisable models. Such problems arise not only due to human factors (such as the subjectivity of annotators, their age, fatigue and stress) but also due to the fuzziness of the meaning associated with various labels related to human behavior. The issue becomes even more prominent when the task is temporal, as it renders the labeling procedure vulnerable to temporal lags caused by varying response times of annotators. Considering that in many of the aforementioned problems the annotation lies in a continuous real space (as opposed to discrete labels), the subjectivity of the annotators becomes much more difficult to model and fuse into a single "ground truth".

- M. A. Nicolaou is with the Department of Computing, Imperial College London, London SW7 2AZ, U.K. E-mail: mihalis@imperial.ac.uk.

- V. Pavlovic is with the Department of Computer Science, Rutgers University, Piscataway, NJ 08854 USA.E-mail:vladimir@cs.rutgers.edu.

- M. Pantic is with the Department of Computing, Imperial College London, London SW7 2AZ, U.K. and also with the EEMCS, University of Twente, Twente, The Netherlands.E-mail: m.pantic@imperial.ac.uk.

Manuscript received 14 Dec. 2012; revised 31 Oct. 2013; accepted 27 Nov. 2013. Date of publication 8 Jan. 2014; date of current version 13 June 2014. Recommended for acceptance by F. de la Torre.

For information on obtaining reprints of this article, please send e-mail to: reprints@ieee.org, and reference the Digital Object Identifier below.

Digital Object Identifier 10.1109/TPAMI.2014.16
A recent emerging trend in affective computing is the adoption of real-valued, continuous dimensional emotion descriptions for learning tasks [1]. The space consists of various dimensions such as valence (ranging from unpleasant to pleasant) and arousal (from relaxed to aroused). In this description, each emotional state is mapped to a point in the dimensional space, thus overcoming the limitation of confining in a small set of discrete classes (such as the typically used six basic emotion classes). In this way, the expressiveness of the description is extended to non-basic emotions, typically manifested in everyday life (e.g., boredom). Nevertheless, the annotation of such data, although performed by multiple trained experts, results in labels which exhibit an amalgam of the aforementioned issues ([2], Fig. 1), leading researchers to adopt solutions based on simple (or weighted) averaging, reliance on a single annotator or quantising the continuous space and thus shifting the problem to the discrete domain (see [3]-[5]), where several solutions have been proposed (see [6]).

A state-of-the-art approach in fusing multiple continuous annotations that can be applied to emotion descriptions is proposed by Raykar et al. [7]. In this work, each noisy annotation is considered to be generated by a Gaussian distribution with the mean being the true label and the variance representing the annotation noise.

A main drawback of [7] lies in the assumption that temporal correspondences of samples are known. One way to find such arbitrary temporal correspondences is via time warping. A state-of-the-art approach for time warping, Canonical Time Warping (CTW) [8], combines Dynamic Time Warping (DTW) and Canonical Correlation Analysis (CCA) with the 


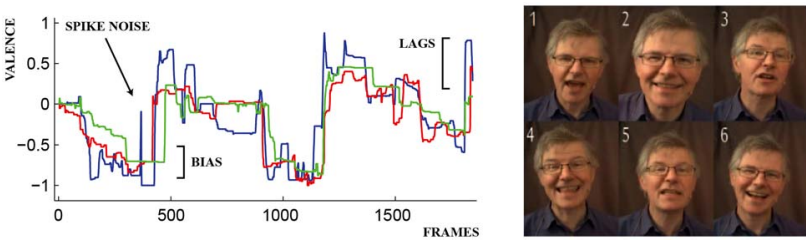

Fig. 1. Valence annotations along with video stills.

aim of aligning a pair of sequences of both different duration and different dimensionality. CTW accomplishes this by simultaneously finding the most correlated features and samples among the two sequences, both in feature space and time. This task is reminiscent of the goal of fusing expert annotations. However, CTW does not directly yield the prototypical sequence, which is considered as a common, denoised and fused version of multiple experts' annotations. As a consequence, this renders neither of the two state-of-the-art methods applicable to our setting.

The latter observation precisely motivates our work; inspired by Probabilistic Canonical Correlation Analysis (PCCA) [9], we initially present the first generalisation of PCCA to learning temporal dependencies in the shared/individual spaces (Dynamic PCCA, DPCCA). By further augmenting DPCCA with time warping, the resulting model (Dynamic PCCA with Time Warpings, DPCTW) can be seen as a unifying framework, concisely applied to both problems. The individual contributions of this work can be summarised as follows:

- In comparison to state-of-the-art approaches in both fusion of multiple annotations and sequence alignment, our model bears several advantages. We assume that the "true" annotation/sequence lies in a shared latent space. E.g., in the problem of fusing multiple emotion annotations, we know that the experts have a common training in annotation. Nevertheless, each carries a set of individual factors which can be assumed to be uninteresting (e.g., annotator/sequence specific bias). In the proposed model, individual factors are accounted for within an annotator-specific latent space, thus effectively preventing the contamination of the shared space by individual factors. Most importantly, we introduce latent-space dynamics which model temporal dependencies in both common and individual signals. Furthermore, due to the probabilistic and dynamic nature of the model, each annotator/sequence's uncertainty can be estimated for each sample, rather than for each sequence.

- In contrast to current work on fusing multiple annotations, we propose a novel framework able to handle temporal tasks. In addition to introducing dynamics, we also employ temporal alignment in order to eliminate temporal discrepancies amongst the annotations.

- We present an elegant extension of DTW-based sequence alignment techniques (e.g., Canonical Time Warping, CTW) to a probabilistic multiple-sequence setting. We accomplish this by treating the problem in a generative probabilistic setting, both in the static (multiset PCCA) and dynamic case (Dynamic PCCA).
The rest of the paper is organised as follows. In Section 2, we describe PCCA and present our extension to multiple sequences. In Section 3, we introduce our proposed Dynamic PCCA, which we subsequently extend with latent space time-warping (DPCTW) as described in Section 4. In Section 5, we introduce two supervised variants of DPCTW which incorporate inputs in a generative (Section 5.1) and discriminative (Section 5.2) manner, while in Section 6 we present an algorithm based on the proposed family of models which ranks and filters annotators. In Section 7, we present various experiments on both synthetic (Section 7.1) and real (Sections 7.2 and 7.3) experimental data, emphasising the advantages of the proposed methods on both the fusion of multiple annotations and sequence alignment.

\section{Multiset Probabilistic CCA}

We consider the probabilistic interpretation of CCA, introduced by Bach \& Jordan [10] and generalised by Klami \& Kaski [9] ${ }^{1}$. In this section, we present an extended version of PCCA [9] (multiset PCCA ${ }^{2}$ ) which is able to handle any arbitrary number of sets. We consider a collection of datasets $\mathcal{D}=\left\{\mathbf{X}_{1}, \mathbf{X}_{2}, \ldots, \mathbf{X}_{N}\right\}$, with each $\mathbf{X}_{i} \in \mathbb{R}^{D_{i} \times T}$ where $D_{i}$ is the dimensionality and $T$ the number of instances. By adopting the generative model for PCCA, the observation sample $n$ of set $\mathbf{X}_{i} \in \mathcal{D}$ is assumed to be generated as

$$
\mathbf{x}_{i, n}=f\left(\mathbf{z}_{n} \mid \mathbf{W}_{i}\right)+g\left(\mathbf{z}_{i, n} \mid \mathbf{B}_{i}\right)+\epsilon_{i},
$$

where $\mathbf{Z}_{i}=\left[\mathbf{z}_{i, 1}, \ldots, \mathbf{z}_{i, T}\right] \in \mathbb{R}^{d_{i} \times T}$ and $\mathbf{Z}=\left[\mathbf{z}_{1}, \ldots, \mathbf{z}_{T}\right] \in$ $\mathbb{R}^{d \times T}$ are the independent latent variables that capture the set-specific individual characteristics and the shared signal amongst all observation sets, respectively. $f($.$) and g($.$) are$ functions that transform each of the latent signals $\mathbf{Z}$ and $\mathbf{Z}_{i}$ into the observation space. They are parametrised by $\mathbf{W}_{i}$ and $\mathbf{B}_{i}$, while the noise for each set is represented by $\epsilon_{i}$, with $\epsilon_{i} \perp \epsilon_{j}, i \neq j$. Similarly to [9], $\mathbf{z}_{n}, \mathbf{z}_{i, n}$ and $\epsilon_{i}$ are considered to be independent (both over the set and the sequence) and normally distributed:

$$
\mathbf{z}_{n}, \mathbf{z}_{i, n} \sim \mathcal{N}(0, \mathbf{I}), \epsilon_{i} \sim \mathcal{N}\left(0, \sigma_{n}^{2} \mathbf{I}\right) .
$$

By considering $f$ and $g$ to be linear functions we have $f\left(\mathbf{z}_{n} \mid \mathbf{W}_{i}\right)=\mathbf{W}_{i} \mathbf{z}_{n}$ and $g\left(\mathbf{z}_{i, n} \mid \mathbf{B}_{i}\right)=\mathbf{B}_{i} \mathbf{z}_{i, n}$, transforming the model presented in Eq. 1, to

$$
\mathbf{x}_{i, n}=\mathbf{W}_{i} \mathbf{z}_{n}+\mathbf{B}_{i} \mathbf{z}_{i, n}+\epsilon_{i} .
$$

Learning the multiset PCCA can be accomplished by generalising the EM algorithm presented in [9], applied to two or more sets. Firstly, $P\left(\mathcal{D} \mid \mathbf{Z}, \mathbf{Z}_{1}, \ldots, \mathbf{Z}_{N}\right)$ is marginalised over set-specific factors $\mathbf{Z}_{1}, \ldots, \mathbf{Z}_{N}$ and optimised on each $\mathbf{W}_{i}$. This leads to the generative model $P\left(\mathbf{x}_{i, n} \mid \mathbf{z}_{n}\right) \sim \mathcal{N}\left(\mathbf{W}_{i} \mathbf{z}_{n}, \Psi_{i}\right)$, where $\Psi_{i}=\mathbf{B}_{i} \mathbf{B}_{i}^{T}+\sigma_{i}^{2} \mathbf{I}$. Subsequently, $P\left(\mathcal{D} \mid \mathbf{Z}, \mathbf{Z}_{1}, \ldots, \mathbf{Z}_{N}\right)$ is marginalised over the common factor $\mathbf{Z}$ and then optimised on each $\mathbf{B}_{i}$ and $\sigma_{i}$. When generalising the algorithm for more than two sets, we also have to consider how to (i) obtain the expectation of the latent space and (ii) provide stable variance updates for all sets.

1. [9] is also related to Tucker's inter-battery factor analysis [11], [12]

2. In what follows we refer to multiset PCCA as PCCA. 
Two quantities are of interest regarding the latent space estimation. The first is the common latent space given one set, $\mathbf{Z} \mid \mathbf{X}_{i}$. In the classical CCA this is analogous to finding the canonical variables [9]. We estimate the posterior of the shared latent variable $\mathbf{Z}$ as follows:

$$
\begin{aligned}
P\left(\mathbf{z}_{n} \mid \mathbf{x}_{i, n}\right) & \sim \mathcal{N}\left(\boldsymbol{\gamma}_{i} \mathbf{x}_{i, n}, \mathbf{I}-\gamma_{i} \mathbf{W}_{i}\right), \\
\boldsymbol{\gamma}_{i} & =\mathbf{W}_{i}^{T}\left(\mathbf{W}_{i} \mathbf{W}_{i}^{T}+\Psi_{i}\right)^{-1} .
\end{aligned}
$$

The latent space given the $n$-th sample from all sets in $\mathcal{D}$, which provides a better estimate of the shared signal manifested in all observation sets is estimated as

$$
\begin{array}{r}
P\left(\mathbf{z}_{n} \mid \mathbf{x}_{1: N, n}\right) \sim \mathcal{N}\left(\boldsymbol{\gamma} \mathbf{x}_{1: N, n}, \mathbf{I}-\boldsymbol{\gamma} \mathbf{W}\right), \\
\boldsymbol{\gamma}=\mathbf{W}^{T}\left(\mathbf{W} \mathbf{W}^{T}+\Psi\right)^{-1},
\end{array}
$$

while the matrices $\mathbf{W}, \Psi$ and $\mathbf{X}_{n}$ are defined as $\mathbf{W}^{T}=$ $\left[\mathbf{W}_{1}^{T}, \mathbf{W}_{2}^{T}, \ldots, \mathbf{W}_{n}^{T}\right], \Psi$ as the block diagonal matrix of $\Psi_{i=1: N}{ }^{3}$ and $\mathbf{x}_{1: N, n}^{T}=\left[\mathbf{x}_{1, n}^{T}, \mathbf{x}_{2, n}^{T}, \ldots, \mathbf{x}_{1: N, n}^{T}\right]$. Finally, the variance is recovered on the full model, $x_{i, n} \sim \mathcal{N}\left(\mathbf{W}_{i} \mathbf{z}_{n}+\right.$ $\left.\mathbf{B}_{i} \mathbf{z}_{i, n}, \sigma_{i}^{2} \mathbf{I}\right)$, as

$$
\begin{aligned}
\sigma_{i}^{2} & =\operatorname{tr}\left(\mathbf{S}-\mathbf{X} \mathbb{E}\left[\mathbf{Z}^{T} \mid \mathbf{X}\right] \mathbf{C}^{T}\right. \\
& \left.-\mathbf{C E}[\mathbf{Z} \mid \mathbf{X}] \mathbf{X}^{T}-\mathbf{C E}\left[\mathbf{Z Z} \mathbf{Z}^{T} \mid \mathbf{X}\right] \mathbf{C}^{T}\right)_{i} \frac{T}{D_{i}},
\end{aligned}
$$

where $\mathbf{S}$ is the sample covariance matrix, $\mathbf{B}$ is the block diagonal matrix of $\mathbf{B}_{i=1: N}, \mathbf{C}=[\mathbf{W}, \mathbf{B}]$, while the subscript $i$ in Eq. 6 refers to the i-th block of the full covariance matrix. Finally, we note that the computational complexity of PCCA for each iteration is similar to deterministic CCA (cubic in the dimensionalities of the datasets and linear in the number of samples). PCCA though also recovers the private space.

\section{DYNAMIC PCCA (DPCCA)}

The PCCA model described in Section 2 exhibits several advantages when compared to the classical formulation of CCA, mainly by providing a probabilistic estimation of a latent space shared by an arbitrary collection of datasets along with explicit noise and private space estimation. Nevertheless, static models are unable to learn temporal dependencies which are very likely to exist when dealing with real-life problems. In fact, dynamics are deemed essential for successfully performing tasks such as emotion recognition, AU detection etc. [13].

Motivated by the former observation, we propose a dynamic generalisation of the static PCCA model introduced in the previous section, where we now treat each $\mathbf{X}_{i}$ as a temporal sequence. For simplicity of presentation, we introduce a linear model ${ }^{4}$ where Markovian dependencies are learnt in the latent spaces $\mathbf{Z}$ and $\mathbf{Z}_{i}$. In other words, the variable $\mathbf{Z}$ models the temporal, shared signal amongst all observation sequences, while $\mathbf{Z}_{i}$ captures the temporal, individual characteristics of each sequence. It is easy to observe that such a model fits perfectly with the problem of fusing multiple annotations, as it does not only capture the temporal shared signal of all annotations, but also models the unwanted, annotator-specific factors over time. Essentially,

3. For brevity of notation, we use $1: N$ to indicate elements $[1, \ldots, N]$, e.g., $\mathbf{X}_{1: N} \equiv\left[\mathbf{X}_{1}, \mathbf{X}_{2}, \ldots, \mathbf{X}_{N}\right]$

4. A non-linear DPCCA model can be derived as in [14], [15]. instead of directly applying the doubly independent priors to $\mathbf{Z}$ as in Eq. 2, we now use the following:

$$
\begin{gathered}
p\left(\mathbf{z}_{t} \mid \mathbf{z}_{t-1}\right) \sim \mathcal{N}\left(\mathbf{A}_{z} \mathbf{z}_{t-1}, \mathbf{V}_{Z}\right), \\
p\left(\mathbf{z}_{i, t} \mid \mathbf{z}_{i, t-1}\right) \sim \mathcal{N}\left(\mathbf{A}_{z_{i}} \mathbf{z}_{i, t-1}, \mathbf{V}_{Z_{i}}\right), n=1, \ldots, N,
\end{gathered}
$$

where the transition matrices $\mathbf{A}_{z}$ and $\mathbf{A}_{z_{i}}$ model the latent space dynamics for the shared and sequence-specific space respectively. Thus, idiosyncratic characteristics of dynamic nature appearing in a single sequence can be accurately estimated and prevented from contaminating the estimation of the shared signal.

The resulting model bears similarities with traditional Linear Dynamic System (LDS) models (e.g. [16]) and the so-called Factorial Dynamic Models, see [17]. Along with Eq. 7,8 and noting Eq. 3, the dynamic, generative model for DPCCA ${ }^{5}$ can be described as

$$
\mathbf{x}_{i, t}=\mathbf{W}_{i, t} \mathbf{z}_{t}+\mathbf{B}_{i} \mathbf{z}_{i, t}+\epsilon_{i}, \epsilon_{i} \sim \mathcal{N}\left(0, \sigma_{i}^{2} \mathbf{I}\right),
$$

where the subscripts $i$ and $t$ refer to the $i$-th observation sequence timestep $t$ respectively.

\subsection{Inference}

To perform inference, we reduce the DPCCA model to a LDS $^{6}$. This can be accomplished by defining a joint space $\hat{\mathbf{Z}}^{T}=\left[\mathbf{Z}^{T}, \mathbf{Z}_{1}^{T}, \ldots, \mathbf{Z}_{N}^{T}\right], \hat{\mathbf{Z}} \in \mathbb{R}^{\hat{d} \times T}$ where $\hat{d}=d+\sum_{i}^{N} d_{i}$ with parameters $\boldsymbol{\theta}=\left\{\mathbf{A}, \mathbf{W}, \mathbf{B}, \mathbf{V}_{\hat{z}}, \hat{\Sigma}\right\}$. Dynamics in this joint space are described as $\mathbf{X}_{t}=[\mathbf{W}, \mathbf{B}] \hat{\mathbf{Z}}_{t}+\boldsymbol{\epsilon}, \hat{\mathbf{Z}}_{t}=\mathbf{A} \hat{\mathbf{Z}}_{t-1}+\mathbf{u}$, where the noise processes $\boldsymbol{\epsilon}$ and $\mathbf{u}$ are defined as

$$
\begin{aligned}
& \boldsymbol{\epsilon} \sim \mathcal{N}(0, \underbrace{\left[\begin{array}{ccc}
\sigma_{1}^{2} \mathbf{I} & & \\
& \ddots & \\
& & \sigma_{N}^{2} \mathbf{I}
\end{array}\right]}_{\hat{\Sigma}}), \\
& \mathbf{u} \sim \mathcal{N}(0, \underbrace{\left[\begin{array}{llll}
\mathbf{V}_{z} & & & \\
& \mathbf{V}_{z_{1}} & \\
& & \ddots & \\
& & & \mathbf{V}_{z_{N}}
\end{array}\right]}_{\mathbf{V}_{\hat{z}}}),
\end{aligned}
$$

where $\mathbf{V}_{z} \in \mathbb{R}^{d \times T}$ and $\mathbf{V}_{z_{i}} \in \mathbb{R}^{d_{i} \times T}$. The other matrices used above are defined as $\mathbf{X}^{T}=\left[\mathbf{X}_{1}^{T}, \ldots, \mathbf{X}_{N}^{T}\right]$, $\mathbf{W}^{T}=\left[\mathbf{W}_{1}^{T}, \ldots, \mathbf{W}_{N}^{T}\right], \mathbf{B}$ as the block diagonal matrix of $\left[\mathbf{B}_{1}, \ldots, \mathbf{B}_{N}\right]$ and $\mathbf{A}$ as the block diagonal matrix of $\left[\mathbf{A}_{z}, \mathbf{A}_{z_{1}}, \ldots, \mathbf{A}_{z_{N}}\right]$. Similarly to LDS, the joint log-likelihood function of DPCCA is defined as

$$
\begin{aligned}
\ln P(\mathbf{X}, \mathbf{Z} \mid \theta) & =\ln P\left(\hat{\mathbf{z}}_{1} \mid \mu, V\right)+\sum_{t=2}^{T} \ln P\left(\hat{\mathbf{z}}_{t} \mid \hat{\mathbf{z}}_{t-1}, \mathbf{A}, \mathbf{V}_{\hat{z}}\right) \\
& +\sum_{t=1}^{T} \ln P\left(\mathbf{x}_{t} \mid \hat{\mathbf{z}}_{t}, \mathbf{W}, \mathbf{B}, \hat{\Sigma}\right)
\end{aligned}
$$

5. The model of Raykar et al. [7] can be considered as a special case of (D)PCCA by setting $\mathbf{W}=\mathbf{I}, \mathbf{B}=\mathbf{0}$ (and disregarding dynamics).

6. For more details on LDS, please see [16] and [18], Chapter 13. 
In order estimate the latent spaces, we apply the RauchTung-Striebel (RTS) smoother on $\hat{\mathbf{Z}}$ (the algorithm can be found in [16], A.3). In this way, we obtain $\mathbb{E}\left[\hat{\mathbf{z}}_{t} \mid \mathbf{X}^{T}\right], V\left[\hat{\mathbf{z}}_{t} \mid \mathbf{X}^{T}\right]$ and $V\left[\hat{\mathbf{z}}_{t} \hat{\mathbf{z}}_{t-1} \mid \mathbf{X}^{T}\right]^{7}$.

\subsection{Parameter Estimation}

The parameter estimation of the M-step has to be derived specifically for this factorised model. We consider the expectation of the joint model log-likelihood (Eq. 12) wrt. posterior and obtain the partial derivatives of each parameter for finding the stationary points. Note the $\mathbf{W}$ and $\mathbf{B}$ matrices appear in the likelihood as:

$$
\begin{gathered}
\mathbb{E}_{\hat{z}}[\ln P(\mathbf{X}, \hat{\mathbf{Z}})]=-\frac{T}{2} \ln |\hat{\Sigma}|-\mathbb{E}_{\hat{z}}\left[\sum_{t=1}^{T}\left(\mathbf{x}_{t}-[\mathbf{W}, \mathbf{B}] \hat{\mathbf{z}}_{t}\right)^{T}\right. \\
\left.\hat{\Sigma}^{-1}\left(\mathbf{x}_{t}-[\mathbf{W}, \mathbf{B}] \hat{\mathbf{z}}_{t}\right)\right]+\ldots
\end{gathered}
$$

Since they are composed of individual $\mathbf{W}_{i}$ and $\mathbf{B}_{i}$ matrices (which are parameters for each sequence $i$ ), we calculate the partial derivatives $\partial \mathbf{W}_{i}$ and $\partial \mathbf{B}_{i}$ in Eq. 13. Subsequently, by setting to zero and re-arranging, we obtain the update equations for each $\mathbf{W}_{i}^{*}$ and $\mathbf{B}_{i}^{*}$ :

$$
\begin{aligned}
\mathbf{W}_{i}^{*} & =\left(\sum_{t=1}^{T} \mathbf{x}_{i, t} \mathbb{E}\left[\mathbf{z}_{i, t}\right]-\mathbf{B}_{i}^{*} \mathbb{E}\left[\mathbf{z}_{i, t} \mathbf{z}_{t}^{T}\right]\right)\left(\sum_{t=1}^{T} \mathbb{E}\left[\mathbf{z}_{t} \mathbf{z}_{t}^{T}\right]\right)^{-1} \\
\mathbf{B}_{i}^{*} & =\left(\sum_{t=1}^{T} \mathbf{x}_{i, t} \mathbb{E}\left[\mathbf{z}_{t}^{T}\right]-\mathbf{W}_{i}^{*} \mathbb{E}\left[\mathbf{z}_{t} \mathbf{z}_{i, t}^{T}\right]\right)\left(\sum_{t=1}^{T} \mathbb{E}\left[\mathbf{z}_{i, t} \mathbf{z}_{i, t}^{T}\right)^{-1}\right.
\end{aligned}
$$

Note that the weights are coupled and thus the optimal solution should be found iteratively. As can be seen, in contrast to PCCA, in DPCCA the individual factors of each sequence are explicitly estimated instead of being marginalised out. Similarly, the transition weight updates for the individual factors $\mathbf{Z}_{i}$ are as follows:

$$
\mathbf{A}_{z, i}^{*}=\left(\sum_{t=2}^{T} E\left[\mathbf{z}_{i, t} \mathbf{z}_{i, t-1}^{T}\right]\right)\left(\sum_{t=2}^{T} E\left[\mathbf{z}_{i, t-1} \mathbf{z}_{i, t-1}^{T}\right]\right)^{-1}
$$

where by removing the subscript $i$ we obtain the updates for $\mathbf{A}_{z}$, corresponding to the shared latent space $\mathbf{Z}$. Finally, the noise updates $\mathbf{V}_{\hat{Z}}$ and $\hat{\Sigma}$ are estimated similarly to LDS [16].

\section{DPCCA WITH TIME WARPINGS}

Both PCCA and DPCCA exhibit several advantages in comparison to the classical formulation of CCA. Mainly, as we have shown, (D)PCCA can inherently handle more than two sequences, building upon the multiset nature of PCCA. This is in contrast to the classical formulation of CCA, which due to the pairwise nature of the correlation operator

7. We note that the complexity of RTS is cubic in the dimension of the state space. Thus, when estimating high dimensional latent spaces, computational or numerical issues may arise (due to the inversion of large matrices). If any of the above is a concern, the complexity of RTS can be reduced to quadratic [19], while inference can be performed more efficiently similarly to [17]. is limited to two sequences ${ }^{8}$. This is crucial for the problems at hand since both methods yield an accurate estimation of the underlying signals of all observation sequences, free of individual factors and noise. However, both PCCA and DPCCA carry the assumption that the temporal correspondences between samples of different sequences are known, i.e. that the annotation of expert $i$ at time $t$ directly corresponds to the annotation of expert $j$ at the same time. Nevertheless, this assumption is often violated since different experts exhibit different time lags in annotating the same process (e.g., Fig. 1, [21]). Motivated by the latter, we extend the DPCCA model to account for this misalignment of data samples by introducing a latent warping process into DPCCA, in a manner similar to [8]. In what follows, we firstly describe some basic background on time-warping and subsequently proceed to define our model.

\subsection{Time Warping}

Dynamic Time Warping (DTW) [22] is an algorithm for optimally aligning two sequences of possibly different lengths. Given sequences $\mathbf{X} \in \mathbb{R}^{D \times T_{x}}$ and $\mathbf{Y} \in \mathbb{R}^{D \times T_{y}}$, DTW aligns the samples of each sequence by minimising the sum-ofsquares cost, i.e. $\left\|\mathbf{X} \Delta_{x}-\mathbf{Y} \Delta_{y}\right\|_{F}^{2}$, where $\Delta_{x} \in \mathbb{R}^{T_{x} \times T_{\Delta}}$ and $\Delta_{y} \in \mathbb{R}^{T_{y} \times T_{\Delta}}$ are binary selection matrices, with $T_{\Delta}$ the aligned, common length. In this way, the warping matrices $\Delta$ effectively re-map the samples of each sequence. Although the number of possible alignments is exponential in $T_{x} T_{y}$, employing dynamic programming can recover the optimal path in $\mathcal{O}\left(T_{x} T_{y}\right)$. Furthermore, the solution must satisfy the boundary, continuity and monotonicity constraints, effectively restricting the space of $\Delta_{x}, \Delta_{y}$ [22].

An important limitation of DTW is the inability to align signals of different dimensionality. Motivated by the former, CTW [8] combines CCA and DTW, thus alowing the alignment of signals of different dimensionality by projecting into a common space via CCA. The optimisation function now becomes $\left\|\mathbf{V}_{x}^{T} \mathbf{X} \Delta_{x}-\mathbf{V}_{y}^{T} \mathbf{Y} \Delta_{y}\right\|_{F}^{2}$, where $\mathbf{X} \in \mathbb{R}^{D_{x} \times T_{x}}, \mathbf{Y} \in \mathbb{R}^{D_{y} \times T_{x}}$, and $\mathbf{V}_{x}, \mathbf{V}_{y}$ are the projection operators (matrices).

\subsection{DPCTW Model}

We define DPCTW based on the graphical model presented in Fig. 2. Given a set $\mathcal{D}$ of $N$ sequences of varying duration, with each sequence $\mathbf{X}_{i}=\left[\mathbf{x}_{i, 1}, \ldots, \mathbf{x}_{i, T_{i}}\right] \in \mathbb{R}^{D_{i} \times T_{i}}$, we postulate the latent common Markov process $\mathbf{Z}=\left\{\mathbf{z}_{1}, \ldots, \mathbf{z}_{t}\right\}$. Firstly, $\mathbf{Z}$ is warped using the warping operator $\Delta_{i}$, resulting in the warped latent sequence $\zeta_{i}$. Subsequently, each $\zeta_{i}$ generates each observation sequence $\boldsymbol{X}_{i}$, also considering the annotator/sequence bias $\mathbf{Z}_{i}$ and the observation noise $\sigma_{i}^{2}$. We note that we do not impose parametric models for warping processes. Inference in this general model can be prohibitively expensive, in particular because of the need to handle the unknown alignments. We instead propose to handle the inference in two steps: (i) fix the alignments $\Delta_{i}$ and find the latent $\mathbf{Z}$ and $\mathbf{Z}_{i}$ 's, and (ii) given the estimated $\mathbf{Z}, \mathbf{Z}_{i}$ find the optimal warpings $\Delta_{i}$. For this, we propose to

8. The recently proposed multiset-CCA [20] can handle multiple sequences but requires maximising over sums of pairwise operations. 

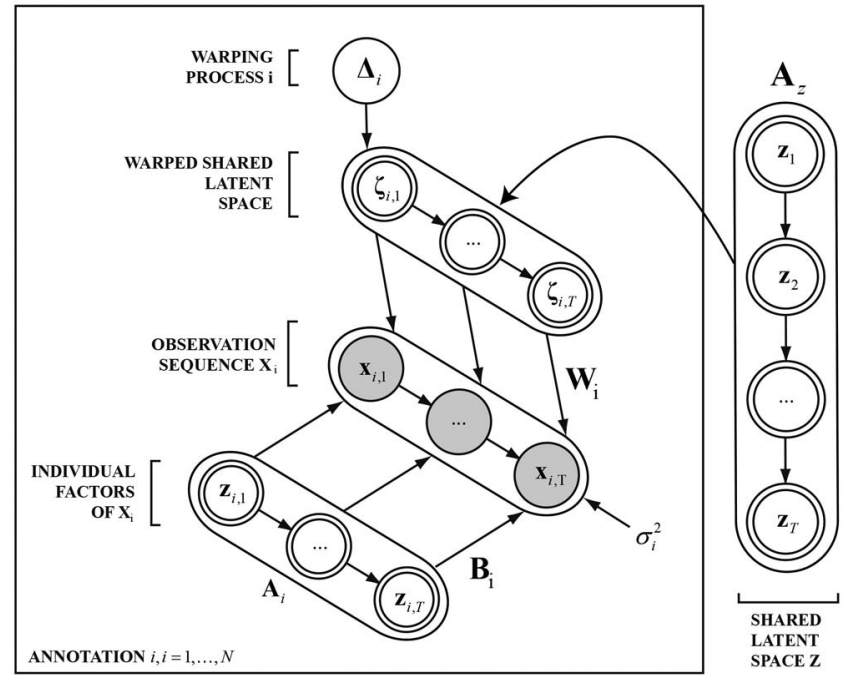

Fig. 2. Graphical model of DPCTW. Shaded nodes represent the observations. By ignoring the temporal dependencies, we obtain the PCTW model.

optimise the following objective function:

$$
\mathcal{L}_{(\mathrm{D}) \mathrm{PCTW}}=\sum_{i}^{N} \sum_{j, j \neq i}^{N} \frac{\left\|\mathbb{E}\left[\mathbf{Z} \mid \mathbf{X}_{i}\right] \Delta_{i}-\mathbb{E}\left[\mathbf{Z} \mid \mathbf{X}_{j}\right] \Delta_{j}\right\|_{F}^{2}}{N(N-1)}
$$

where when using PCCA, $\mathbb{E}\left[\mathbf{Z} \mid \mathbf{X}_{i}\right]=\mathbf{W}_{i}^{T}\left(\mathbf{W}_{i} \mathbf{W}_{i}^{T}+\Psi_{i}\right)^{-1} \mathbf{X}_{i}$ (Eq. 4). For DPCCA, $\mathbb{E}\left[\mathbf{Z} \mid \mathbf{X}_{i}\right]$ is inferred via RTS smoothing (Section 3). A summary of the full algorithm is presented in Algorithm 1.

At this point, it is important to clarify that our model is flexible enough to be straightforwardly used with varying warping techniques. For example, the Gauss-Newton warping proposed in [23] can be used as the underlying warping process for DPCCA, by replacing the projected data $\mathbf{V}_{i}^{T} \mathbf{X}_{i}$ with $\mathbb{E}\left[\mathbf{Z} \mid \mathbf{X}_{i}\right]$ in the optimisation function. Algorithmically, this only changes the warping process (line 3, Algorithm 1). Finally, we note that since our model iterates between estimating the latent spaces with (D)PCCA and warping, the computational complexity of time warping is additive to the cost of each iteration. In case of the DTW alignment for two sequences, this incurs an extra cost of $O\left(T_{x} T_{y}\right)$. In case of more than two sequences, we utilise a DTW-based algorithm, which is a variant of the so-called Guide Tree Progressive Alignment, since the complexity of dynamic programming increases exponentially with the number of sequences. Similar algorithms are used in state-of-the-art sequence alignment software in biology, e.g., Clustar [24]. The complexity of the employed algorithm is $O\left(N^{2} T_{\max }^{2}\right)$ where $T_{\max }$ is the maximum (aligned) sequence length and $N$ the number of sequences. More efficient implementations can also be used by employing various constraints [22].

\section{Features for annotator Fusion}

In the previous sections, we considered the observed data to consist only of the given annotations, $\mathcal{D}=\left\{\mathbf{X}_{1}, \ldots, \mathbf{X}_{N}\right\}$. Nevertheless, in many problems one can extract additional observed information, which we can consider as a form of complementary input (e.g., visual or audio features). In fact,

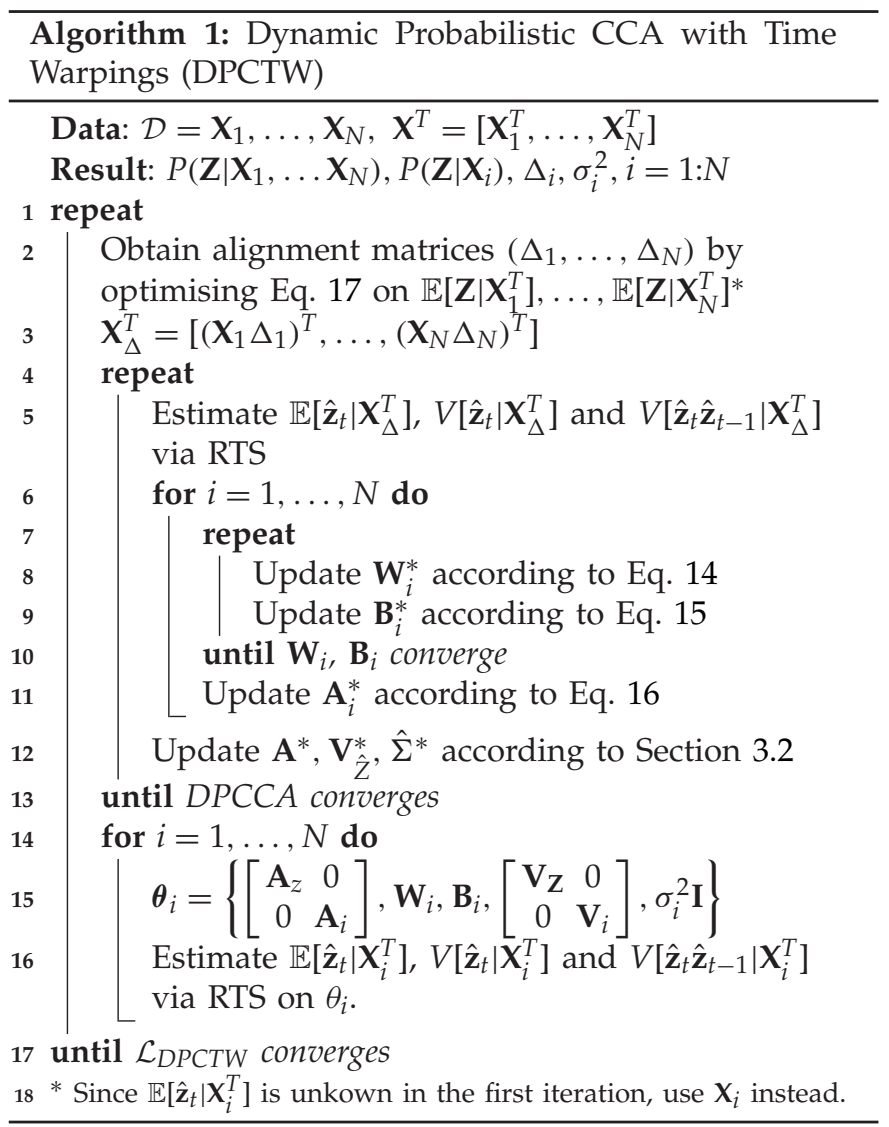

in problems where annotations are subjective and no objective ground truth is available for any portion of the data, such input can be considered as the only objective reference to the annotation/sequence at hand. Thus, incorporating it into the model can significantly aid the determination of the ground truth.

Motivated by the latter argument, we propose two models which augment DPCCA/DPCTW with inputs. Since the family of component analysis techniques we study are typically unsupervised, incorporating inputs leads to a form of supervised learning. Such models can find a wide variety of applications since they are able to exploit label information in addition to observations. A suitable example lies in dimensional affect analysis, where it has been shown that specific emotion dimensions correlate better with specific cues, (e.g., valence with facial features, arousal with audio features [1], [4]). Thus, one can know a-priori which features to use for specific annotations.

Throughout this discussion, we assume that a set of complementary input or features $\mathbf{Y}=\left\{\mathbf{Y}_{1}, \ldots, \mathbf{Y}_{v}\right\}$ is available, where $\mathbf{Y}_{j} \in \mathbb{R}^{D_{y_{j}} \times T_{y_{j}}}$. While discussing extensions of DPCCA, we assume that all sequences have equal length. When incorporating time warping, sequences can have different lengths.

\subsection{Supervised-Generative DPCCA (SG-DPCCA)}

We firstly consider the model where we simply augment the observation model with a set of features $\mathbf{Y}_{j}$. In this case, the generative model for DPCCA (Eq. 9) is:

$$
\mathbf{x}_{i, t}=\mathbf{W}_{i, t} \mathbf{z}_{t}+\mathbf{B}_{i} \mathbf{z}_{i, t}+\epsilon_{i},
$$




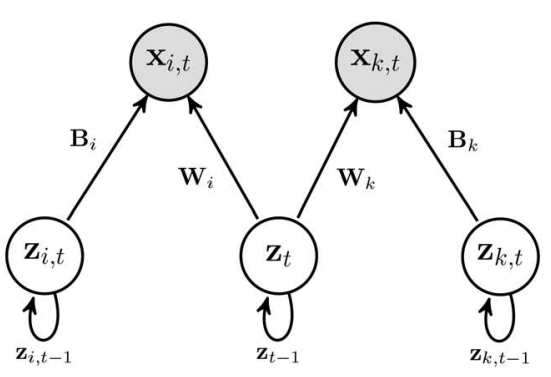

(a)

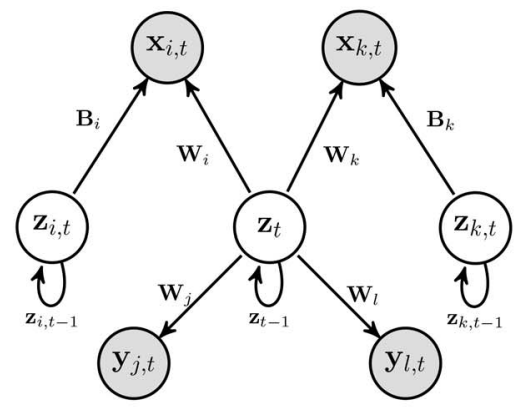

(b)

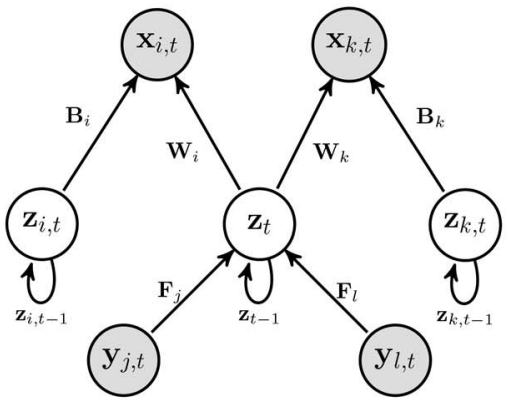

(c)

Fig. 3. Comparing the model structure of DPCCA (a) to SG-DPCCA, and (b) SD-DPCCA. (c) Notice that the shared space $\mathbf{z}$ generates both observations and features in SG-DPCCA, while in SD-DPCCA, the shared space at time $t$ is generated by regressing from the features $y$ and the previous shared space state $\mathbf{z}_{t-1}$.

$$
\mathbf{y}_{j, t}=h_{j, s}\left(\mathbf{z}_{t} \mid \mathbf{W}_{j, t}\right)+h_{j, p}\left(\mathbf{z}_{j, t} \mid \mathbf{B}_{j}\right)+\epsilon_{j},
$$

where $i=\{1, \ldots, N\}$ and $j=\{N+1, \ldots, N+v+1\}$. The arbitrary functions $h$ map the shared space to the feature space in a generative manner, while $\epsilon_{j} \sim \mathcal{N}\left(0, \sigma_{j}^{2} \mathbf{I}\right)$. The latent priors are still defined as in Eq. 7,8. By assuming that $h$ is linear, we can group the parameters $\mathbf{W}=\left[\mathbf{W}_{1}, \ldots, \mathbf{W}_{N}, \ldots, \mathbf{W}_{N+v}\right], \mathbf{B}$ as the block diagonal of $\left(\left[\mathbf{B}_{1}, \ldots, \mathbf{B}_{N}, \ldots, \mathbf{B}_{N+v}\right]\right)$ and $\hat{\boldsymbol{\Sigma}}$ as the block diagonal of $\left(\left[\sigma^{2} \mathbf{I}_{1}, \ldots, \sigma^{2} \mathbf{I}_{N}, \ldots, \sigma^{2} \mathbf{I}_{N+v}\right]\right)$. Inference is subsequently applied as described in Section 3.

This model, which we dub SG-DPCCA, in effect captures a common shared space of both annotations $\mathbf{X}$ and available features $\mathbf{Y}$ for each sequence. In our generative scenario, the shared space generates both features and annotations. By further setting $h_{j, p}$ to zero, one can force the representation of the entire feature space $\mathbf{Y}_{j}$ onto the shared space, thus imposing stronger constraints on the shared space given each annotation $\mathbf{Z} \mid \mathbf{X}_{i}$. As we will show, this model can help identify unwanted annotations by simply analysing the posteriors of the shared latent space. We note that the additional form of supervision imposed by the input on the model is reminiscent of SPCA for PCA [25]. The discriminative ability added by the inputs (or labels) also relates DPCCA to LDA [10]. The graphical model of SG-DPCCA is illustrated in Fig. 3(b).

SG-DPCCA can be easily extended to handle timewarping as described in Section 4 for DPCCA (SGDPCTW). The main difference is that now one would have to introduce one more warping function for each set of features, resulting in a set of $N+v$ functions. Denoting the complete data/input set as $\mathcal{D}^{o}=\left\{\mathbf{X}_{1}, \ldots, \mathbf{X}_{N}, \mathbf{Y}_{1}, \ldots, \mathbf{Y}_{\nu}\right\}$, the objective function for obtaining the time warping functions $\Delta_{i}$ for SG-DPCTW can be defined as:

$$
\mathcal{L}_{S D P C T W^{o}}=\sum_{i}^{N+v} \sum_{j, j \neq i}^{N+v} \frac{\left\|\mathbb{E}\left[\mathbf{Z} \mid \mathcal{D}_{i}^{o}\right] \Delta_{i}-\mathbb{E}\left[\mathbf{Z} \mid \mathcal{D}_{j}^{o}\right] \Delta_{j}\right\|_{F}^{2}}{(N+v)(N+v-1)} .
$$

\subsection{Supervised-Discrimative DPCCA (SD-DPCCA)}

The second model augments the DPCCA model by regressing on the given features. In this case, the posterior of the shared space (Eq. 7) is formulated as

$$
p\left(\mathbf{z}_{t} \mid \mathbf{z}_{t-1}, \mathbf{Y}_{1: v}, \mathbf{A}, \mathbf{V}_{\hat{z}}\right) \sim
$$

$$
\mathcal{N}\left(\mathbf{A}_{z} \mathbf{z}_{t-1}+\sum_{j=1}^{v} h_{j}\left(\mathbf{Y}_{j} \mid \mathbf{F}_{j}\right), \mathbf{V}_{z}\right)
$$

where each function $h_{j}$ performs regression on the features $\mathbf{Y}_{j}$, while $\mathbf{F}_{j} \in \mathbb{R}^{d \times D_{y_{j}}}$ are the loadings for the features (where the latent dimensionality is $d$ ). This is similar to how input is modelled in a standard LDS [15]. To find the parameters, we maximise the complete-data likelihood (Eq. 12), where we replace the second term referring to the latent probability with Eq. 21,

$$
\sum_{t=2}^{T} \ln P\left(\hat{\mathbf{z}}_{t} \mid \hat{\mathbf{z}}_{t-1}, \mathbf{Y}_{1: v}, \mathbf{A}, \mathbf{V}_{\hat{z}}\right) \text {. }
$$

In this variation, the shared space at step $t$ is generated from the previous latent state $\mathbf{z}_{t-1}$ as well as the features at step $t-1, \sum_{j=1}^{v} \mathbf{y}_{j, t-1}$ (Fig. 3(c)). We dub this model SDDPCCA. Without loss of generality we assume $h$ is linear, i.e. $h_{j, s}=\mathbf{W}_{j, t} \mathbf{z}_{t}$, while we model the feature signal only in the shared space, i.e. $h_{j, p}=0$. Finding the saddle points of the derivatives with respect to the parameters yields the following updates for the matrices $\mathbf{A}_{z}$ and $\mathbf{F}_{j}, \forall j=1, \ldots, v$ :

$$
\begin{gathered}
\mathbf{A}_{z}^{*}=\left(\sum_{t=2}^{T} E\left[\mathbf{z}_{t} \mathbf{z}_{t-1}^{T}\right]-\sum_{j=1}^{\nu} \mathbf{F}_{j}^{*} \mathbf{y}_{j, t}\right)\left(\sum_{t=2}^{T} E\left[\mathbf{z}_{t-1} \mathbf{z}_{t-1}^{T}\right]\right)^{-1}, \\
\mathbf{F}_{j}^{*}=\left(\mathbb{E}\left[\mathbf{z}_{t}\right]-\mathbf{A}_{z}^{*} \mathbb{E}\left[\mathbf{z}_{t-1}\right]-\sum_{i=1, i \neq j}^{\nu} \mathbf{F}_{i}^{*} \mathbf{Y}_{i}\right) \mathbf{Y}_{j}^{-1}
\end{gathered}
$$

Note that as with the loadings on the shared/individual spaces ( $\mathbf{W}$ and $\mathbf{B}$ ), the optimisation of $\mathbf{A}_{z}$ and $\mathbf{F}_{j}$ matrices should again be determined recursively. Finally, the estimation of $\mathbf{V}_{\mathbf{Z}}$ also changes accordingly:

$$
\begin{aligned}
\mathbf{V}_{\mathbf{z}}^{*}= & \frac{1}{T-1} \sum_{t=2}^{T}\left(\mathbb{E}\left[\mathbf{z}_{t} \mathbf{z}_{t}^{T}\right]-\mathbb{E}\left[\mathbf{z}_{t} \mathbf{z}_{t-1}^{T}\right] \mathbf{A}_{z}^{* T}\right. \\
& -\mathbf{A}_{z}^{*} \mathbb{E}\left[\mathbf{z}_{t-1} \mathbf{z}_{t}^{T}\right]+\mathbf{A}_{z}^{*} \mathbb{E}\left[\mathbf{z}_{t-1} \mathbf{z}_{t-1}^{T}\right] \mathbf{A}_{z}^{* T} \\
& +\sum_{j=1}^{v}\left(\mathbf{A}_{z}^{*} \mathbb{E}\left[\mathbf{z}_{t-1}\right] \mathbf{Y}_{j}^{* T} \mathbf{F}_{j}^{* T}+\mathbf{F}_{j}^{*} \mathbf{Y}_{j} \mathbb{E}\left[\mathbf{z}_{t-1}^{T}\right] \mathbf{A}_{z}^{* T}\right. \\
& +\mathbf{F}_{j}^{*} \mathbf{Y}_{j} \sum_{i=1, i \neq j}^{v} \mathbf{Y}_{i}^{T} \mathbf{F}_{i}^{* T}-\mathbb{E}\left[\mathbf{z}_{t}\right] \mathbf{Y}_{j}^{T} \mathbf{F}_{j}^{* T} \\
& \left.\left.-\mathbf{F}_{j}^{*} \mathbf{Y}_{j} \mathbb{E}\left[\mathbf{z}_{t}^{T}\right]\right)\right) .
\end{aligned}
$$

SD-DPCCA can be straight-forwardly extended with time-warping as with DPCCA in Section 4, resulting in SD-DPCTW. Another alignment step is required before performing the recursive updates mentioned above in order 
to find the correct training/testing pairs for $\mathbf{z}_{t}$ and $\mathbf{Y}$. Assuming the warping matrices are $\boldsymbol{\Delta}_{z}$ and $\boldsymbol{\Delta}_{y}$, then in Eq. $23 \mathbf{z}$ is replaced with $\boldsymbol{\Delta}_{z} \mathbf{z}$ and $\mathbf{y}$ with $\boldsymbol{\Delta}_{y} \mathbf{y}$. The influence of features $\mathbf{Y}$ on the shared latent space $\mathbf{Z}$ in SD-DPCCA and SG-DPCCA is visualised in Fig. 3.

\subsection{Varying Dimensionality}

Typically, we would expect the dimensionality of a set of annotations to be the same. Nevertheless in certain problems, especially when using input features as in SG-DPCCA (Section 5.1), this is not the case. Therefore, in case the observations/input features are of varying dimensionalities, one can scale the third term of the likelihood (Eq. 12) in order to balance the influence of each sequence during learning regardless of its dimensionality:

$$
\begin{array}{r}
\sum_{t=1}^{T}\left(\sum_{j=1}^{v} \frac{1}{D_{y_{j}}} \ln \left(P\left(\mathbf{y}_{t, j} \mid \hat{\mathbf{z}}_{t}, \mathbf{W}_{j}, \mathbf{B}_{j}, \sigma_{j}^{2}\right)\right)+\right. \\
\left.\sum_{j=1}^{N} \frac{1}{D_{i}} \ln \left(P\left(\mathbf{x}_{t, j} \mid \hat{\mathbf{z}}_{t}, \mathbf{W}_{j}, \mathbf{B}_{j}, \sigma_{i}^{2}\right)\right)\right) .
\end{array}
$$

\section{Ranking and Filtering AnNotations}

In this section, we will refer to the issue of ranking and filtering available annotations. Since in general, we consider that there is no "ground truth" available, it is not an easy task to infer which annotators should be discarded and which kept. A straightforward option would be to keep the set of annotators which exhibit a decent level of agreement with each other. Nevertheless, this naive criterion will not suffice in case where e.g., all the annotations exhibit moderate correlation, or where sets of annotations are clustered in groups which are intra-correlated but not inter-correlated.

The question that naturally arises is how to rank and evaluate the annotators when there is no ground truth available and their inter-correlation is not helpful. We remind that DPCCA maximises the correlation of the annotations in the shared space $\mathbf{Z}$, by removing bias, temporal discrepancies and other nuisances from each annotation. It would therefore be reasonable to expect the latent posteriors for each annotation $\left(\mathbf{Z} \mid \mathbf{X}_{i}\right)$, to be as close as possible. Furthermore, the closer the posterior given each annotation $\left(\mathbf{Z} \mid \mathbf{X}_{i}\right)$ to the posterior given all sequences $(\mathbf{Z} \mid \mathcal{D})$, the higher the ranking of the annotator should be, since the closer it is, the larger the portion of the shared information is contained in the annotators signal.

The aforementioned procedure can detect spammers, i.e. annotators who do not even pay attention at the sequence they are annotating and adversarial or malicious annotators that provide erroneous annotations due to e.g., a conflict of interests and can rank the confidence that should be assigned to the rest of the annotators. Nevertheless, it does not account for the case where multiple clusters of annotators are intra-correlated but not inter-correlated. In this case, it is most probable that the best-correlated group will prevail in the ground truth determination. Yet, this does not mean that the best-correlated group is the correct one. In this case, we propose using a set of inputs (e.g., tracking facial points), which can essentially represent the "gold

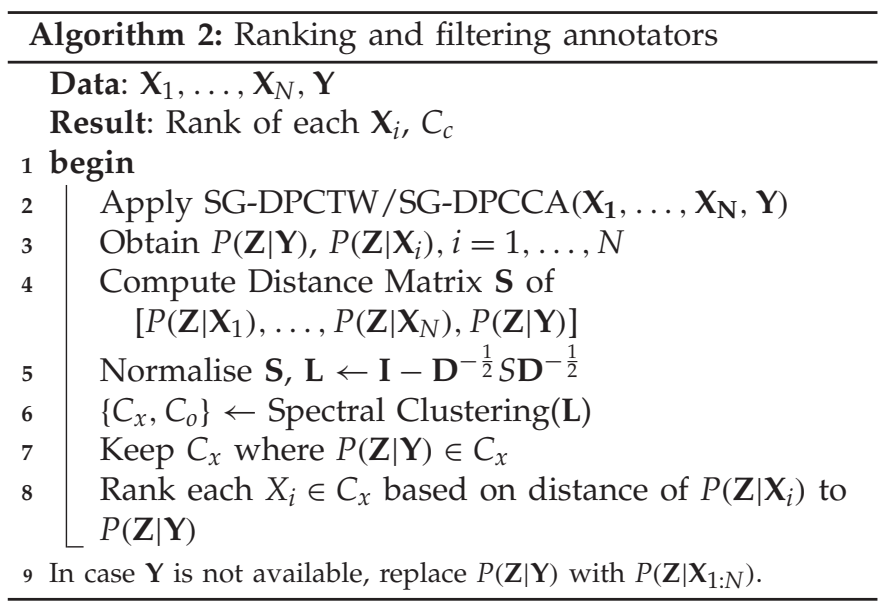

standard". The assumption underlying this proposal is that the correct sequence features should maximally correlate with the correct annotations of the sequence. This can be straightforwardly performed with SG-DPCCA, where we attain $\mathbf{Z} \mid \mathbf{Y}$ (shared space given input) and compare to $\mathbf{Z} \mid \mathbf{X}_{i}$ (shared space given annotation $i$ ).

The comparison of latent posteriors is further motivated by R.J. Aumann's agreement theorem [26]: "If two people are Bayesian rationalists with common priors, and if they have common knowledge of their individual posteriors, then their posteriors must be equal". Since our model maintains the notion of "common knowledge" in the estimation of the shared space, it follows from Aumann's theorem that the individual posteriors $\mathbf{Z} \mid \mathbf{X}_{i}$ of each annotation $i$ should be as close as possible. This is a sensible assumption, since one would expect that if all bias, temporal discrepancies and other nuisances are removed from annotations, then there is no rationale for the posteriors of the shared space to differ.

A simple algorithm for filtering/ranking annotations (utilising spectral clustering [27]) can be found in Algorithm 2. The goal of the algorithm is to find two clusters, $C_{x}$ and $C_{0}$, containing (i) the set of annotations which are correlated with the ground truth, and (ii) the set of "outlier" annotations, respectively. Firstly, DPCCA/DPCTW is applied. Subsequently, a similarity/distance matrix is constructed based on the posterior distances of each annotation $\mathbf{Z} \mid \mathbf{X}_{i}$ along with the features $\mathbf{Z} \mid \mathbf{Y}$. By performing spectral clustering, one can keep the cluster to which $\mathbf{Z} \mid \mathbf{Y}$ belongs $\left(C_{x}\right)$ and disregard the rest of the annotations belonging in $C_{0}$. The ranking of the annotators is computed implicitly via the distance matrix, as it is the relative distance of each $\mathbf{Z} \mid \mathbf{X}_{i}$ to $\mathbf{Z} \mid \mathbf{Y}$. In other words, the feature posterior is used here as the "ground truth". Depending on the application (or in case features are not available), one can use the posterior given all annotations, $\mathbf{Z} \mid \mathbf{X}_{1}, \ldots, \mathbf{X}_{N}$ instead of $\mathbf{Z} \mid \mathbf{Y}$. Examples of distances/metrics that can be used include the alignment error (see Section 4) or the KL divergence between normal distributions (which can be made symmetric by employing e.g., the Jensen-Shannon divergence, i.e. $\left.D_{J S}(P \| Q)=\frac{1}{2} D_{K L}(P \| Q)+\frac{1}{2} D_{K L}(Q \| P)\right)$.

We note that in case of irrelevant or malicious annotations, we assume that the corresponding signals will be 


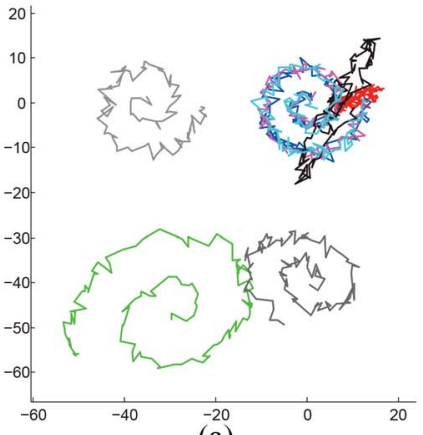

(a)

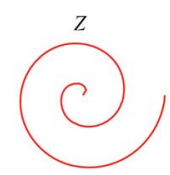

(b)

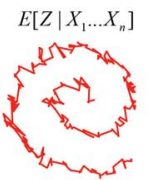

(d)

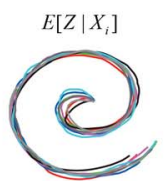

(c)

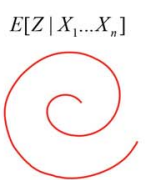

(e)

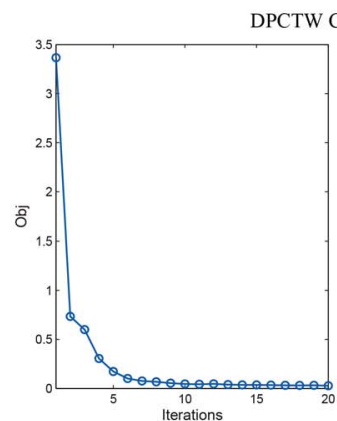

(f)

Fig. 4. Noisy synthetic experiment. (a) Initial, noisy time series. (b) True latent signal from which the noisy, transformed spirals where attained in (a). (c) Alignment achieved by DPCTW. The shared latent space recovered by (d) PCTW and (e) DPCTW. (f) Convergence of DPCTW in terms of the objective (Obj) (Eq. 17) and the path difference between the estimated alignment and the true alignment path (PDGT).

moved to the private space and will not interfere with the time warping. Nevertheless, in order to ensure this, one can impose constraints on the warping process. This is easily done by modifying the DTW by imposing e.g., slope or global constraints such as the Itakura Parallelogram or the Sakoe-Chiba band, in order to constraint the warping path while also decreasing the complexity (see Chap. 5, of [22]). Furthermore, other heuristics can be applied, e.g. firstly filter out the most irrelevant annotations by applying SGDPCCA without time warping, or threshold the warping objective directly (Eq. 17).

\section{EXPERIMENTS}

In order to evaluate the proposed models, in this section, we present a set of experiments on both synthetic (Section 7.1) and real (Sections 7.2 and 7.3) data.

\subsection{Synthetic Data}

For synthetic experiments, we employ a setting similar to [8]. A set of 2D spirals are generated as $\mathbf{X}_{i}=\mathbf{U}_{i}^{T} \tilde{\mathbf{Z}} \mathbf{M}_{i}^{T}+\mathbf{N}$, where $\tilde{\mathbf{Z}} \in \mathbb{R}^{2 \times T}$ is the true latent signal which generates the $\mathbf{X}_{i}$, while the $\mathbf{U}_{i} \in \mathbb{R}^{2 \times 2}$ and $\mathbf{M}_{i} \in \mathbb{R}^{T_{i} \times m}$ matrices impose random spatial and temporal warping. The signal is furthermore perturbed by additive noise via the matrix $\mathbf{N} \in \mathbb{R}^{2 \times T}$. Each $\mathbf{N}(i, j)=e \times b$, where $e \sim \mathcal{N}(0,1)$ and $b$ follows a Bernoulli distribution with $P(b=1)=1$ for Gaussian and $P(b=1)=0.4$ for spike noise. The length of the synthetic sequences varies, but is approximately 200 .

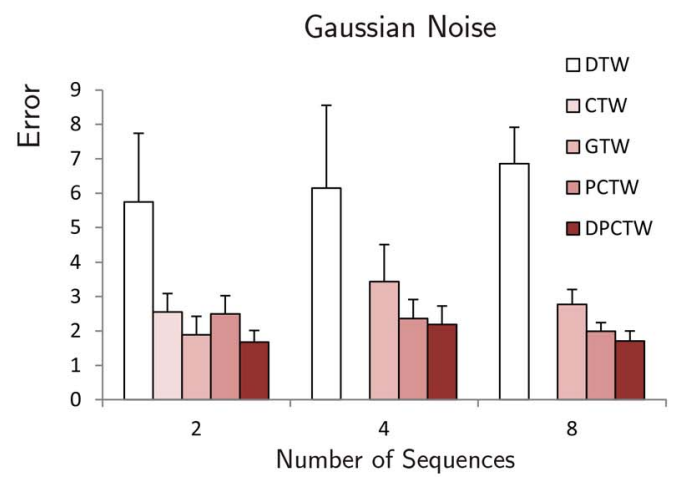

This experiment can be interpreted as both of the problems we are examining. Viewed as a sequence alignment problem the goal is to recover the alignment of each noisy $\mathbf{X}_{i}$, where in this case the true alignment is known. Considering the problem of fusing multiple annotations, the latent signal $\mathbf{Z}$ represents the true annotation while the individual $\mathbf{X}_{i}$ form the set of noisy annotations containing annotation-specific characteristics. The goal is to recover the true latent signal (in DPCCA terms, $\mathbb{E}\left[\mathbf{Z} \mid \mathbf{X}_{1}, \ldots, \mathbf{X}_{N}\right]$ ).

The error metric we used computes the distance from the ground truth alignment $(\tilde{\Delta})$ to the alignment recovered by each algorithm $(\Delta)$ [23], and is defined as:

$$
\begin{aligned}
\operatorname{error} & =\frac{\operatorname{dist}(\Pi, \tilde{\Pi})+\operatorname{dist}(\tilde{\Pi}, \Pi)}{T_{\Delta}+\tilde{T}_{\Delta}}, \\
\operatorname{dist}\left(\Pi_{1}, \Pi_{2}\right) & \left.=\sum_{i=1}^{T_{\Delta}^{1}} \min \left(\left\{\left\|\pi_{1}^{(i)}-\pi_{2}^{(j)}\right\|\right\}\right)_{j=1}^{T_{\Delta}^{2}}\right),
\end{aligned}
$$

where $\Pi_{i} \in \mathbb{R}^{T_{\Delta}^{i} \times N}$ contains the indices corresponding to the binary selection matrices $\boldsymbol{\Delta}_{i}$, as defined in Section 4.1 (and [23]), while $\pi^{(j)}$ refers to the $j$-th row of $\Pi$. For qualitative evaluation, in Fig. 4, we present an example of applying (D)PCTW on 5 sequences. As can be seen, DPCTW is able to recover the true, de-noised, latent signal which generated the noisy observations (Fig. 4(e)), while also aligning the noisy sequences (Fig. 4(c)). Due to the temporal modelling of DPCTW, the recovered latent space is almost identical to the true signal $\tilde{\mathbf{Z}}$ (Fig. 4(b)). PCTW on the other hand is unable to entirely remove the noise (Fig. 4(d)). Fig. 5 shows

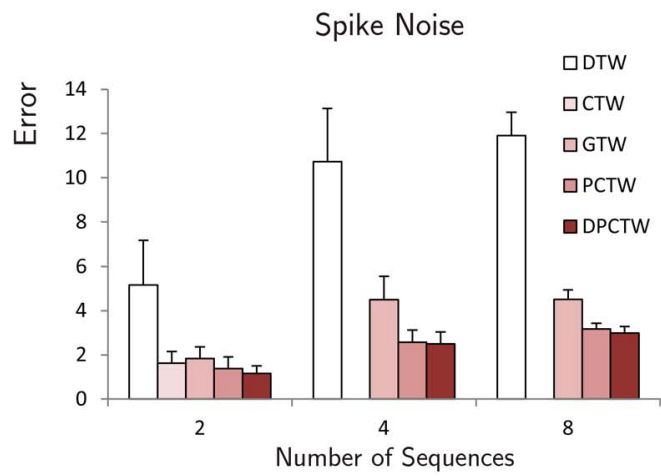

Fig. 5. Synthetic experiment comparing the alignment attained by DTW, CTW, GTW, PCTW and DPCTW on spirals with spiked and Gaussian noise. 


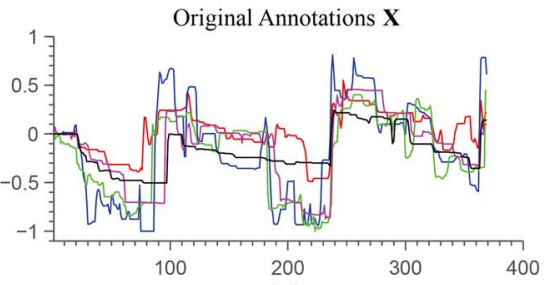

(a)

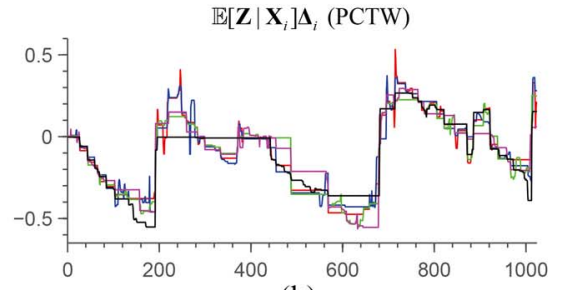

(b)

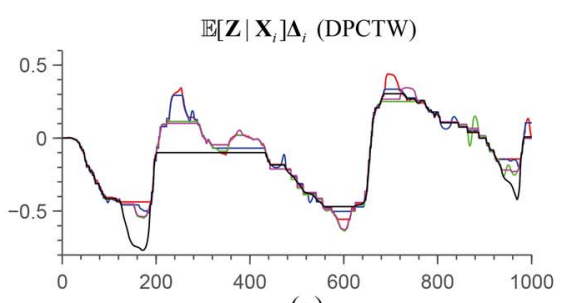

(c)
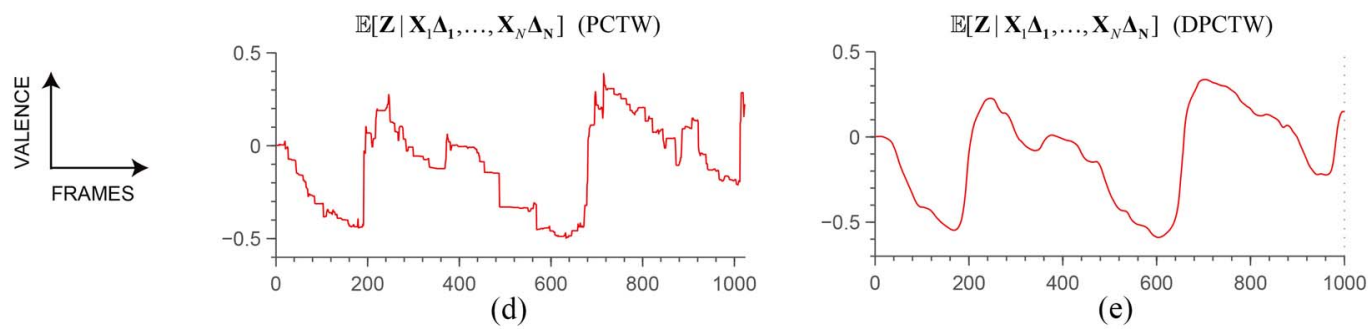

Fig. 6. Applying (D)PCTW to continuous emotion annotations. (a) Original valence annotations from 5 experts. (b, c) Alignment obtained by PCTW and DPCTW respectively, (d, e) Shared space obtained by PCTW and DPCTW respectively, which can be considered as the "derived ground truth".

further results comparing related methods. CTW and GTW perform comparably for two sequences, both outperforming DTW. In general, PCTW seems to perform better than CTW, while DPCTW provides better alignment than other methods compared.

\subsection{Real Data I: Fusing Multiple Annotations}

In order to evaluate (D)PCTW in case of real data, we employ the SEMAINE database [21]. The database contains a set of audio-visual recordings of subjects interacting with operators. Each operator assumes a certain personality - happy, gloomy, angry and pragmatic - with a goal of inducing spontaneous emotions by the subject during a naturalistic conversation. We use a portion of the database containing recordings of 6 different subjects, from over 40 different recording sessions, with a maximum length of 6000 frames per segment. As the database was annotated in terms of emotion dimensions by a set of experts (varying from 2 to 8 ), no single ground truth is provided along with the recordings. Thus, by considering $\mathbf{X}$ to be the set of annotations and applying (D)PCTW, we obtain $\mathbb{E}[\mathbf{Z} \mid \mathcal{D}] \in \mathbb{R}^{1 \times T}$ (given all warped annotations) ${ }^{9}$, which represents the shared latent space with annotator-specific factors and noise removed. We assume that $\mathbb{E}[\mathbf{Z} \mid \mathcal{D}]$ represents the ground truth. An example of this procedure for (D)PCTW can be found in Fig. 6. As can be seen, DPCTW provides a smooth, aligned estimate, eliminating temporal discrepancies, spike-noise and annotator bias. In this experiment, we evaluate the proposed models on four emotion dimensions: valence, arousal, power, and anticipation (expectation).

To obtain features for evaluating the ground truth, we track the facial expressions of each subject via a particle filtering tracking scheme [28]. The tracked points include the corners of the eyebrows (4 points), the eyes (8 points), the nose ( 3 points), the mouth ( 4 points) and the chin ( 1 point), resulting in $202 \mathrm{D}$ points for each frame.

9. We note that latent (D)PCTW posteriors used, e.g. $\mathbf{Z} \mid \mathbf{X}_{i}$ are obtained on time-warped observations, e.g. $\mathbf{Z} \mid \mathbf{X}_{i} \Delta_{\mathbf{i}}$ (see Algorithm 1)
For evaluation, we consider a training sequence $\mathbf{X}$, for which the set of annotations $\mathcal{A}_{x}=\left\{\mathbf{a}_{1}, \ldots, \mathbf{a}_{R}\right\}$ is known. From this set $\left(\mathcal{A}_{x}\right)$, we derive the ground truth $\mathcal{G} \mathcal{T}_{\mathbf{X}}$ - for (D)PCTW, $\mathcal{G T}_{\mathbf{X}}=\mathbb{E}\left[\mathbf{Z} \mid \mathcal{A}_{x}\right]$. Using the tracked points $\mathcal{P}_{\mathbf{X}}$ for the sequence, we train a regressor to learn the function $f_{x}: \mathcal{P}_{\mathbf{X}} \rightarrow \mathcal{G}^{\mathcal{T}} \mathbf{X}$. In (D)PCTW, $\mathcal{P}_{x}$ is firstly aligned with $\mathcal{G} \mathcal{T}_{x}$ as they are not necessarily of equal length. Subsequently given a testing sequence $\mathbf{Y}$ with tracked points $\mathcal{P}_{y}$, using $f_{x}$ we predict each emotion dimension $\left(f_{x}\left(\mathcal{P}_{y}\right)\right)$. The procedure for deriving the ground truth is then applied on the annotations of sequence $\mathbf{Y}$, and the resulting $\mathcal{G} \mathcal{T}_{y}$ is evaluated against $f_{x}\left(\mathcal{P}_{y}\right)$. The correlation coefficient of the $\mathcal{G} \mathcal{T}_{y}$ and $f_{x}\left(\mathcal{P}_{y}\right)$ (after the two signals are temporally aligned) is then used as the evaluation metric for all compared methods.

The reasoning behind this experiment is that the "best" estimation of the ground truth (i.e. the gold standard) should maximally correlate with the corresponding input features - thus enabling any regressor to learn the mapping function more accurately.

We also perform experiments with the supervised variants of DPCTW, i.e. SG-DPCTW and SD-DPCTW. In this case, a set of features $\mathrm{Y}$ is used for inferring the ground truth, $\mathbf{Z} \mid \mathcal{D}$. Since we already used the facial trackings for evaluation, in order to avoid biasing our results ${ }^{10}$, we use features from the audio domain. In particular, we extract a set of audio features consisting of 6 mel-frequency Cepstrum Coefficients (MFCC), 6 MFCC-Delta coefficients along with prosody features (signal energy, root mean squared energy and pitch), resulting in a 15 dimensional feature vector. The audio features are used to derive the ground truth with our supervised models, exactly acting an objective reference to our sequence. In this way, we impose a further constraint on the latent space: it should also explain the audio cues and not only the annotations, given that the two sets are correlated. Subsequently, the procedure

10. Since we use the facial points for evaluating the derived ground truth, if we had also used them for deriving the ground truth we would bias the evaluation procedure. 
TABLE 1

Comparison of Ground Truth Evaluation Based on the Correlation Coefficient (COR), on Session Dependent Experiments. The Standard Deviation over All Results Is Denoted by $\sigma$

\begin{tabular}{rccccccccccccccccccc}
\hline & SD-DPCTW & \multicolumn{1}{c}{ SG-DPCTW } & \multicolumn{2}{c}{ DPCTW } & \multicolumn{2}{c}{ PCTW } & \multicolumn{2}{c}{ DPCCA } & \multicolumn{2}{c}{ PCCA } & RAYKAR [7] & A-AVG \\
\hline & COR & $\boldsymbol{\sigma}$ & COR & $\boldsymbol{\sigma}$ & COR & $\boldsymbol{\sigma}$ & COR & $\boldsymbol{\sigma}$ & COR & $\boldsymbol{\sigma}$ & COR & $\boldsymbol{\sigma}$ & COR & $\boldsymbol{\sigma}$ & COR & $\boldsymbol{\sigma}$ \\
Valence & $\mathbf{0 . 7 8}$ & 0.18 & $\mathbf{0 . 7 8}$ & 0.17 & $\mathbf{0 . 7 7}$ & 0.18 & 0.70 & 0.18 & 0.64 & 0.21 & 0.63 & 0.20 & 0.61 & 0.20 & 0.54 & 0.36 \\
Arousal & 0.75 & 0.18 & $\mathbf{0 . 7 7}$ & 0.19 & $\mathbf{0 . 7 5}$ & 0.22 & 0.64 & 0.22 & 0.63 & 0.23 & 0.63 & 0.26 & 0.60 & 0.25 & 0.42 & 0.41 \\
Power & 0.78 & 0.13 & $\mathbf{0 . 8 5}$ & 0.10 & 0.77 & 0.16 & 0.76 & 0.10 & 0.68 & 0.16 & 0.67 & 0.18 & 0.62 & 0.22 & 0.42 & 0.36 \\
Expectation & 0.82 & 0.09 & $\mathbf{0 . 8 3}$ & 0.10 & 0.78 & 0.11 & 0.75 & 0.16 & 0.68 & 0.16 & 0.74 & 0.17 & 0.62 & 0.20 & 0.48 & 0.40 \\
\hline
\end{tabular}

described above for unsupervised evaluation with facial trackings is employed.

For regression, we employ RVM [29] with a Gaussian kernel. We perform both session-dependent experiments, where the validation was performed on each session separately, and session-independent experiments where different sessions were used for training/testing. In this way, we validate the derived ground truth generalisation ability (i) when the set of annotators is the same and (ii) when the set of annotators may differ.

Session-dependent and session-independent results are presented in Tables 1 and 2. We firstly discuss the unsupervised methods. As can be seen, taking a simple annotator average (A-AVG) gives the worse results (as expected), with a very high standard deviation and weak correlation. The model of Raykar et al. [7] provides better results, which can be justified by the variance estimation for each annotator. Modelling annotator bias and noise with (D)PCCA further improves the results. It is important to note that incorporating alignment is significant for deriving the ground truth; this is reasonable since when the annotations are misaligned, shared information may be modelled as individual factors or vice-versa. Thus, PCTW improves the results further while DPCTW provides the best results, confirming our assumption that combining dynamics, temporal alignment, modelling noise and individual-annotator bias leads to a more objective ground truth. Finally, regarding supervised models SG-DPCTW and SD-DPCTW, we can observe that the inclusion of audio features in the ground truth generation improves the results, with SG-DPCTW providing better correlated results than SD-DPCTW. This is reasonable since in SG-DPCTW the features $\mathbf{Y}$ are explicitly generated from the shared space, thus imposing a form of strict supervision, in comparison to SD-DPCTW where the inputs essentially elicit the shared space.

\subsubsection{Ranking Annotations}

We perform the ranking of annotations as proposed in Algorithm 2 to a set of emotion dimension annotations from the SEMAINE database.
In Fig. 7(a), we illustrate an example where an irrelevant structured annotation (sinusoid), has been added to a set of five true annotations. Obviously the sinusoid can be considered a spammer annotation since essentially, it is independent of the actual sequence at hand. In the figure we can see that (i) the derived ground truth is not affected by the spammer annotation, (ii) the spammer annotation is completely captured in the private space, and (iii) that the spammer annotation is detected in the distance matrix of $\mathbb{E}\left[\mathbf{Z} \mid \mathbf{X}_{i}\right]$ and $\mathbb{E}[\mathbf{Z} \mid \mathbf{X}]$.

In Fig. 7(b), we present an example where a set of 5 annotations has been used along with 8 spammers. The spammers consist of random Gaussian distributions along with structured periodical signals (i.e. sinusoids). We can see that it is difficult to discriminate the spammers by analysing the distance matrix of $\mathbf{X}$ since they do maintain some correlation with the true annotations. By applying Algorithm 2, we obtain the distance matrix of the latent posteriors $\mathbf{Z} \mid \mathbf{X}_{i}$ and $\mathbf{Z} \mid \mathcal{D}$. In this case, we can clearly detect the cluster of annotators which we should keep. By applying spectral clustering, the spammer annotations are isolated in a single cluster, while the shared space along with the true annotations fall into the other cluster. This is also obvious by observing the inferred weight vector $(\mathbf{W})$, which is nearzero for sequences 6-14, implying that the shared signal is ignored when reconstructing the specific annotation (i.e. the reconstruction is entirely from the private space ). Finally, this is also obvious by calculating the KL divergence comparing each individual posterior $\mathbf{Z} \mid X_{i}$ to the shared space posterior given all annotations $\mathbf{Z} \mid \mathcal{D}$, where sequences 6-14 have a high distance while 1-5 have a distance which is very close to zero.

In Fig. 7(c), we present another example where in this case, we joined two sets of annotations which were recorded for two distinct sequences (annotators 1-6 for sequence $\mathrm{A}$ and annotators 7-12 for sequence B). In the distance matrix taken on the observations $\mathbf{X}$, we can see how the two clusters of annotators are already discriminable, with the second cluster, consisting of annotations for sequence $\mathrm{B}$, appearing more correlated. We use the facial

TABLE 2

Comparison of Ground Truth Evaluation Based on the Correlation Coefficient (COR), on Session Independent Experiments. The Standard Deviation over All Results Is Denoted by $\sigma$

\begin{tabular}{|c|c|c|c|c|c|c|c|c|c|c|c|c|c|c|c|c|}
\hline & \multicolumn{2}{|c|}{ SD-DPCTW } & \multicolumn{2}{|c|}{ SG-DPCTW } & \multicolumn{2}{|c|}{ DPCTW } & \multicolumn{2}{|c|}{ PCTW } & \multicolumn{2}{|c|}{ DPCCA } & \multicolumn{2}{|c|}{ PCCA } & \multicolumn{2}{|c|}{ RAYKAR [7] } & \multicolumn{2}{|c|}{ A-AVG } \\
\hline & COR & $\sigma$ & COR & $\sigma$ & COR & $\sigma$ & COR & $\sigma$ & COR & $\sigma$ & COR & $\sigma$ & COR & $\sigma$ & COR & $\sigma$ \\
\hline Valence & 0.73 & 0.19 & 0.73 & 0.19 & 0.72 & 0.22 & 0.66 & 0.24 & 0.62 & 0.28 & 0.58 & 0.23 & 0.57 & 0.27 & 0.53 & 0.33 \\
\hline Arousal & 0.74 & 0.15 & 0.74 & 0.17 & 0.71 & 0.20 & 0.61 & 0.23 & 0.59 & 0.23 & 0.52 & 0.28 & 0.50 & 0.29 & 0.33 & 0.40 \\
\hline Power & 0.72 & 0.28 & 0.75 & 0.24 & 0.72 & 0.34 & 0.70 & 0.19 & 0.60 & 0.26 & 0.58 & 0.27 & 0.57 & 0.27 & 0.39 & 0.31 \\
\hline Expectation & 0.76 & 0.21 & 0.76 & 0.15 & 0.73 & 0.20 & 0.70 & 0.18 & 0.63 & 0.20 & 0.64 & 0.25 & 0.63 & 0.22 & 0.44 & 0.39 \\
\hline
\end{tabular}


(a)

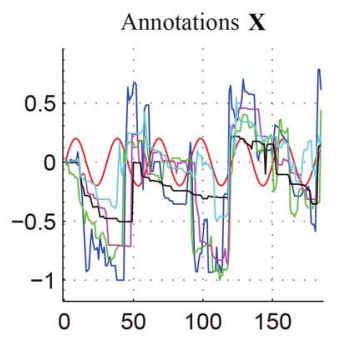

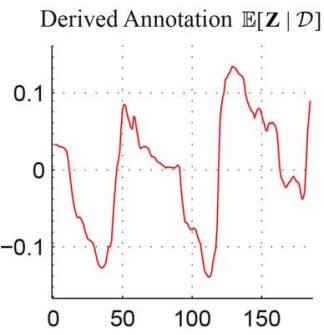
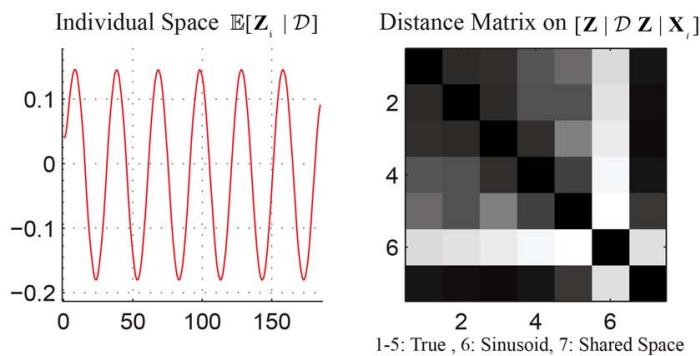

Distance Matrix on $\mathbf{X}$

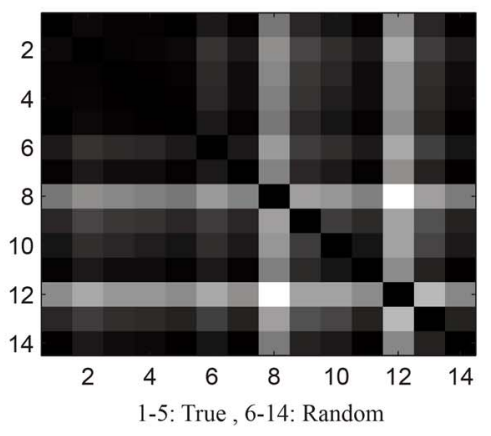

Distance Matrix on $\left[\mathbf{Z}\left|\mathbf{X}_{i} \mathbf{Z}\right| \mathcal{D}\right]$

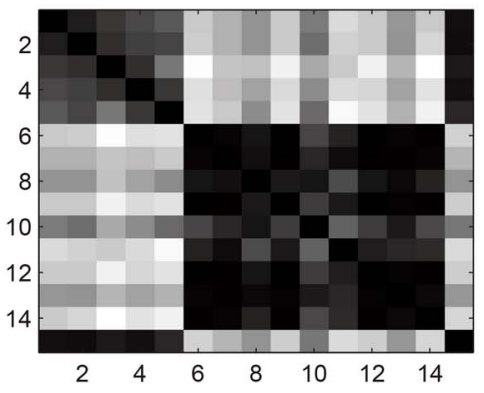

1-5: True, 6-14: Random, 15: Shared Space

Distance Matrix on $\left[\mathbf{Z}\left|\mathbf{X}_{i} \mathbf{Z}\right| \mathbf{Y}\right]$
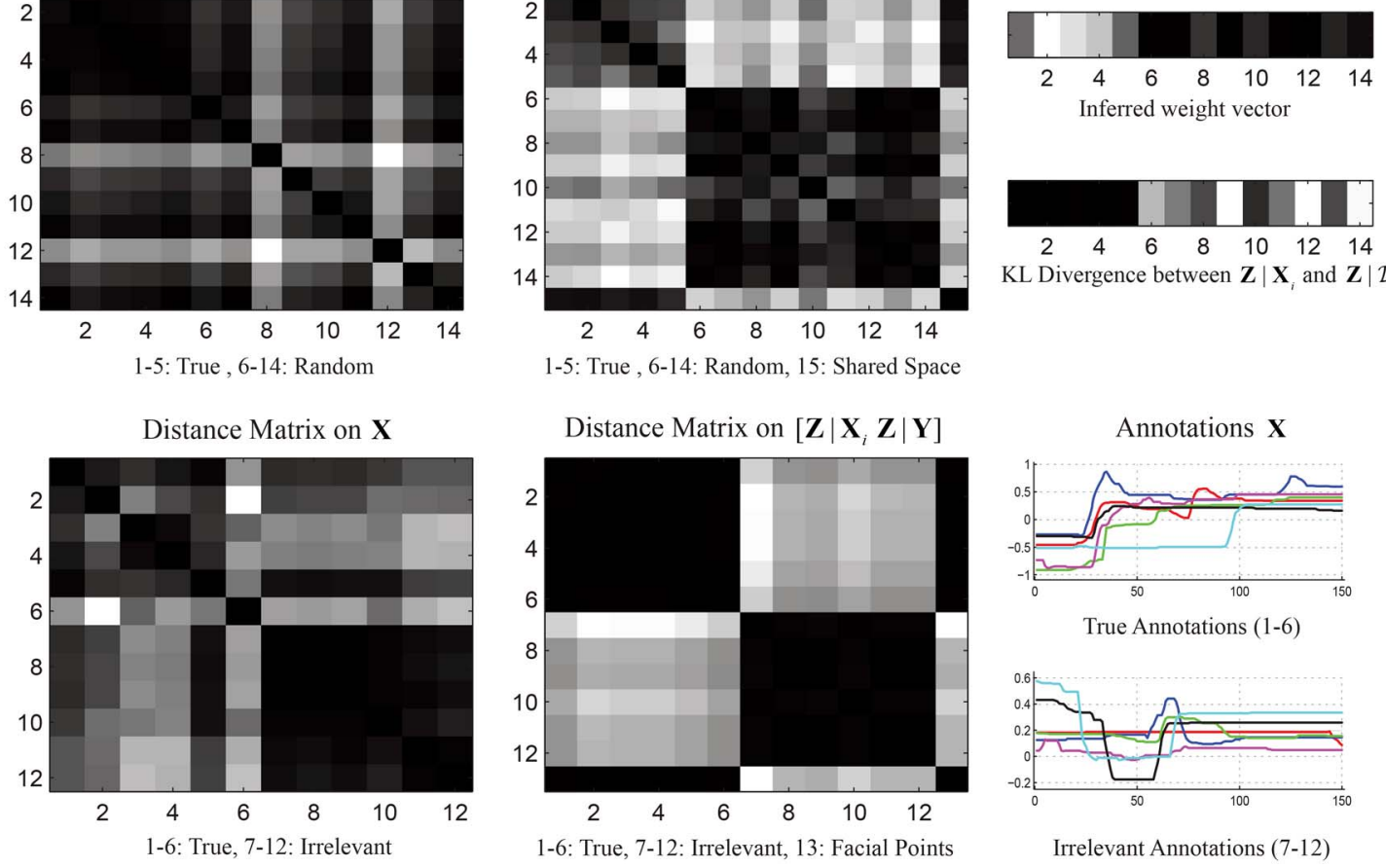

KL Divergence between $\mathbf{Z} \mid \mathbf{X}_{i}$ and $\mathbf{Z} \mid \mathcal{D}$

Annotations X

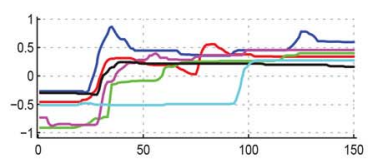

True Annotations (1-6)

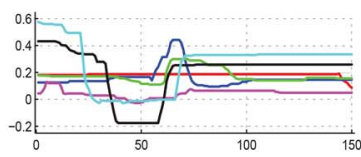

Irrelevant Annotations (7-12)

Fig. 7. Annotation filtering and ranking (black - low, white - high). (a) Experiment with a structured false annotation (sinusoid). The shared space is not affected by the false annotation, which is isolated in the individual space. (b) Experiment with 5 true and 9 spammer (random) annotations. (c) Experiment with 6 true annotations, 7 irrelevant but correlated annotations (belonging to a different sequence). The facial points $Y$, corresponding to the 6 true annotations, were used for supervision (with SG-DPCCA).

trackings for sequence A (tracked as described in this section) as the features $\mathbf{Y}$, and then apply Algorithm 2. As can be seen in the distance matrix of $\left[\mathbf{Z}\left|\mathbf{X}_{i}, \mathbf{Z}\right| \mathbf{Y}\right]$, (i) the two clusters of annotators have been clearly separated, and (ii) the posterior of features $\mathbf{Z} \mid \mathbf{Y}$ clearly is much closer to annotations 1-6, which are the true annotations of sequence A.

\subsection{Real Data II: Action Unit Alignment}

In this experiment we aim to evaluate the performance of (D)PCTW for the temporal alignment of facial expressions. Such applications can be useful for methods which require pre-aligned data, e.g. AAM (Active Appearance Models). For this experiment, we use a portion of the MMI database which contains more than 300 videos, ranging from 100 to 200 frames. Each video is annotated (per frame) in terms of the temporal phases of each Action Unit (AU) manifested by the subject being recorded, namely neutral, onset, apex and offset. For this experiment, we track the facial expressions of each subject capturing $202 \mathrm{D}$ points, as in Section 7.2.
Given a set of videos where the same AU is activated by the subjects, the goal is to temporally align the phases of each AU activation across all videos containing that AU, where the facial points are used as features. In the context of DPCTW, each $\mathbf{X}_{i}$ is the facial points of video $i$ containing the same $\mathrm{AU}$, while $\mathbf{Z} \mid \mathbf{X}_{i}$ is now the common latent space given video $i$, the size of which is determined by cross-validation, and is constant over all experiments for a specific noise level.

In Fig. 8 we present results based on the number of misaligned frames for AU alignment, on all action unit temporal phases (neutral, onset, apex, offset) for AU 12 (smile), on a set of 50 pairs of videos from MMI. For this experiment, we used the facial features relating to the lower face, which consist of $112 \mathrm{D}$ points. The features were perturbed with sparse spike noise in order to simulate the misdetection of points with detection-based trackers, in order to evaluate the robustness of the proposed techniques. Values were drawn from the normal distribution $\mathcal{N}(0,1)$ and added (uniformly) to $5 \%$ of the length of each video. We gradually increased the number of features 

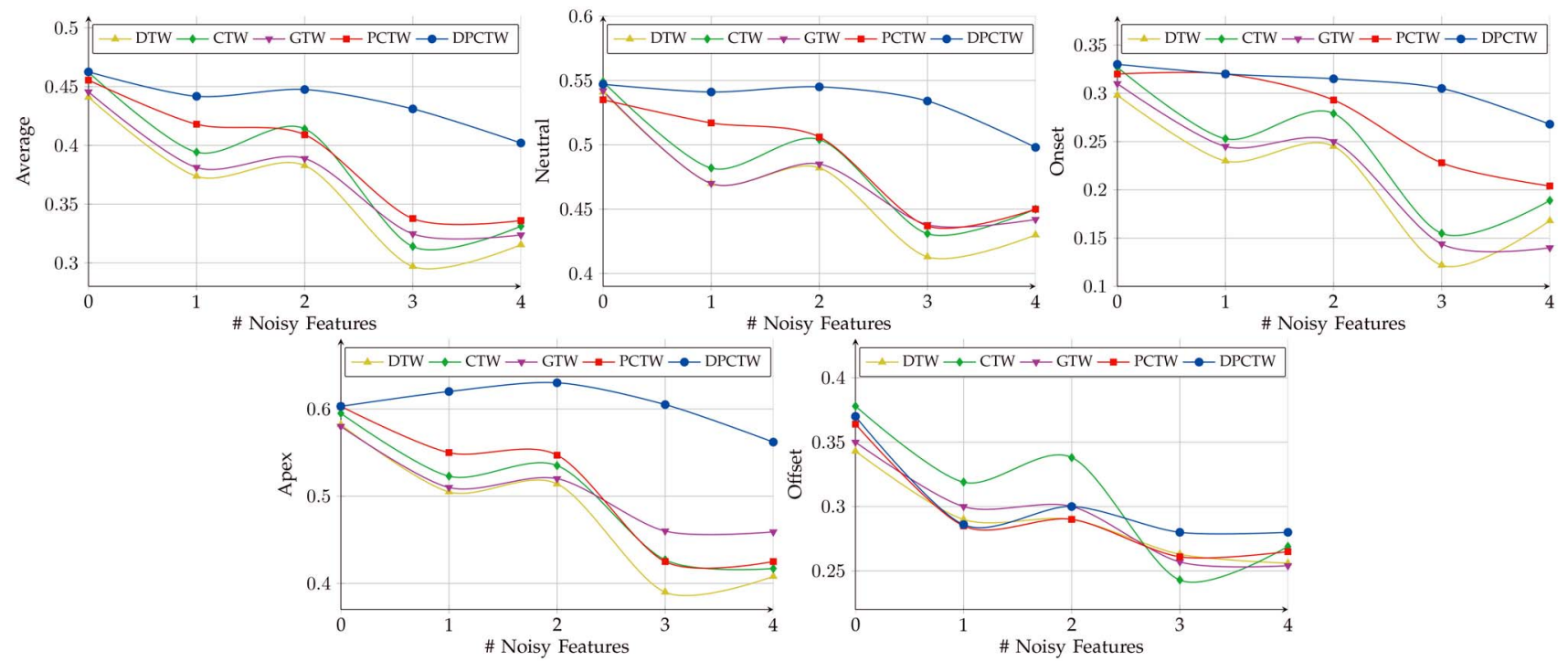

Fig. 8. Accuracy of DTW, CTW, GTW, PCTW and DPCTW on the problem of action unit alignment under spiked noise added to an increasing number of features for $\mathrm{AU}=12$ (smile).

perturbed by noise from 0 to 4 . To evaluate the accuracy of each algorithm, we use a robust, normalised metric. In more detail, let us say that we have two videos, with features $\mathbf{X}_{1}$ and $\mathbf{X}_{2}$, and $\mathrm{AU}$ annotations $\mathcal{A}_{1}$ and $\mathcal{A}_{2}$. Based on the features, the algorithm at hand recovers the alignment matrices $\Delta_{1}$ and $\Delta_{2}$. By applying the alignment matrices on the AU annotations $\left(\mathcal{A}_{1} \Delta_{1}\right.$ and $\left.\mathcal{A}_{2} \Delta_{2}\right)$, we know to which temporal phase of the AU each aligned frame of each video corresponds to. Therefore, for a given temporal phase (e.g., neutral), we have a set of frame indices which are assigned to the specific temporal phase in video $1, P h_{1}$ and video 2 , $P h_{2}$. The accuracy is then estimated as $\frac{P h_{1} \cap P h_{2}}{P h_{1} \cup P h_{2}}$. This essentially corresponds to the ratio of correctly aligned frames to the total duration of the temporal phase accross the aligned videos.

As can be seen in the average results in Fig. 8, the best performance is clearly obtained by DPCTW. It is also interesting to highlight the accuracy of DPCTW on detecting the apex, which essentially is the peak of the expression. This can be attributed to the modelling of dynamics, not only in the shared latent space of all facial point sequences but also in the domain of the individual characteristics of each sequence (in this case identifying and removing the added temporal spiked noise). PCTW peforms better on average compared than CTW and GTW, while the latter two methods perform similarly. It is interesting to note that GTW seems to overpeform CTW and PCTW for aligning the apex of the expression for higher noise levels. Furthermore, we point-out that the Gauss-Newton warping used in GTW is likely to perform better for longer sequences. Example frames from videos showing the unaligned and DPCTW-aligned videos are shown in Fig. 9.

\section{Conclusion}

In this work, we presented DPCCA, a novel, dynamic and probabilistic model based on the multiset probabilistic interpretation of CCA. By integrating DPCCA with time warping, we proposed DPCTW, which can be interpreted as a unifying framework for solving the problems of (i) fusing multiple imperfect annotations and (ii) aligning temporal sequences. Furthermore, we extended DPCCA/DPCTW to a supervised scenario, where one can exploit inputs and observations, both in a discriminative and generative framework. We show that the family of probabilistic models which we present is this paper is able to rank and filter annotators merely by utilising inferred model statistics. Finally, our experiments show that DPCTW features

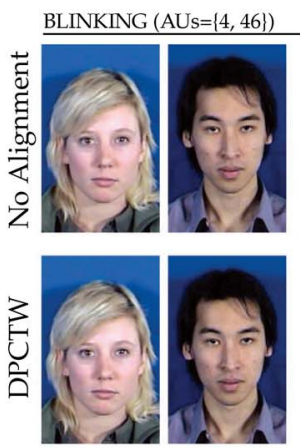

FRAMES
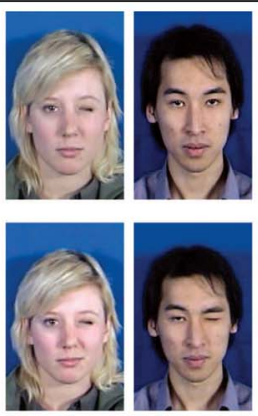

(a)

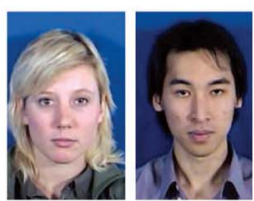

MOUTH OPEN (AUs=\{25, 26\})
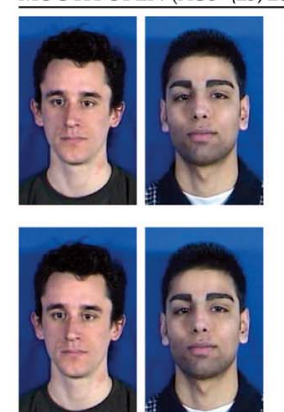

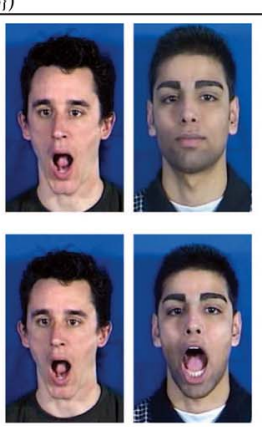

(b)

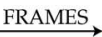
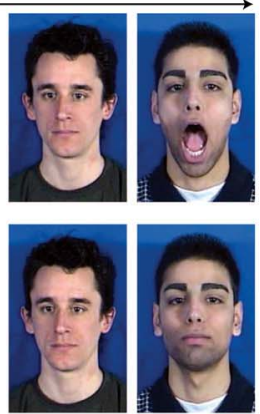

Fig. 9. Example stills from a set of videos from the MMI database, comparing the original videos to the aligned videos obtained via DPCTW under spiked noise on 4 2D points. (a) Blinking, AUs 4 and 46. (b) Mouth open, AUs 25 and 27. 
such as temporal alignment, learning dynamics, identifying individual annotator/sequence factors and incorporating inputs are critical for robust performance of fusion in challenging affective behavior analysis tasks.

\section{ACKNOWLEDGMENT}

This work is supported by the European Research Council under the ERC Starting Grant ERC-2007-StG203143 (MAHNOB), by the European Communitys 7th Framework Programme [FP7/2007-2013] under Grant 288235 (FROG) and by the National Science Foundation under Grant IIS 0916812.

\section{REFERENCES}

[1] H. Gunes, B. Schuller, M. Pantic, and R. Cowie, "Emotion representation, analysis and synthesis in continuous space: A survey," in Proc. IEEE Int. Conf. EmoSPACE WS, Santa Barbara, CA, USA, 2011, pp. 827-834.

[2] R. Cowie and G. McKeown. (2010). Statistical Analysis of Data From Initial Labelled Database and Recommendations for an Economical Coding Scheme [Online]. Available: http://www.semaine-project.eu/

[3] M. Wöllmer et al.,"Abandoning emotion classes," in Proc. INTERSPEECH, 2008, pp. 597-600.

[4] M. A. Nicolaou, H. Gunes, and M. Pantic, "Continuous prediction of spontaneous affect from multiple cues and modalities in valence-arousal space," IEEE Trans. Affect. Comput., vol. 2, no. 2, pp. 92-105, Apr.-Jun. 2011.

[5] M. Grimm, K. Kroschel, E. Mower, and S. Narayanan, "Primitivesbased evaluation and estimation of emotions in speech," Speech Commun., vol. 49, no. 10-11, pp. 787-800, Oct. 2007.

[6] K. Audhkhasi and S. S. Narayanan, "A globally-variant locallyconstant model for fusion of labels from multiple diverse experts without using reference labels," IEEE Trans. Pattern Anal. Mach. Intell., vol. 35, no. 4, pp. 769-783, Apr. 2013.

[7] V. C. Raykar et al., "Learning from crowds," J. Mach. Learn. Res., vol. 11, pp. 1297-1322, Apr. 2010.

[8] F. Zhou and F. De la Torre, "Canonical time warping for alignment of human behavior," in Proc. Adv. NIPS, 2009, pp. 2286-2294.

[9] A. Klami and S. Kaski, "Probabilistic approach to detecting dependencies between data sets," Neurocomput., vol. 72, no. 1-3, pp. 39-46, Dec. 2008.

[10] F. R. Bach and M. I. Jordan, "A probabilistic interpretation of canonical correlation analysis," Univ. California, Berkeley, CA, USA, Tech. Rep. 688, 2006.

[11] L. R. Tucker, "An inter-battery method of factor analysis," Psychometrika, vol. 23, no. 2, pp. 111-136, 1958.

[12] M. W. Browne, "The maximum-likelihood solution in interbattery factor analysis," Br. J. Math. Stat. Psychol., vol. 32, no. 1, pp. 75-86, 1979.

[13] Z. Zeng, M. Pantic, G. I. Roisman, and T. S. Huang, "A survey of affect recognition methods: Audio, visual, and spontaneous expressions," IEEE Trans. Pattern Anal. Mach. Intell., vol. 31, no. 1, pp. 39-58, Jan. 2009.

[14] M. Kim and V. Pavlovic, "Discriminative learning for dynamic state prediction," IEEE Trans. Pattern Anal. Mach. Intell., vol. 31, no. 10, pp. 1847-1861, Oct. 2009.

[15] Z. Ghahramani and S. T. Roweis, "Learning nonlinear dynamical systems using an EM algorithm," in Advances NIPS. Cambridge, MA, USA: MIT Press, 1999, pp. 599-605.

[16] S. Roweis and Z. Ghahramani, "A unifying review of linear Gaussian models," Neural Comput., vol. 11, no. 2, pp. 305-345, 1999.

[17] Z. Ghahramani, M. I. Jordan, and P. Smyth, "Factorial hidden Markov models," in Machine Learning. Cambridge, MA, USA: MIT Press, 1997.

[18] C. M. Bishop, Pattern Recognition and Machine Learning. Secaucus, NJ, USA: Springer-Verlag, Inc., 2006.

[19] R. Van der Merwe and E. Wan, "The square-root unscented Kalman filter for state and parameter-estimation," in Proc. IEEE Int. Conf. Acoustics, Speech, Signal Process., vol. 6. Salt Lake City, UT, USA, 2001, pp. 3461-3464.
[20] M. A. Hasan, "On multi-set canonical correlation analysis," in Proc. Int. Joint Conf. Neural Netw., Piscataway, NJ, USA: IEEE Press, 2009, pp. 2640-2645.

[21] G. McKeown, M. F. Valstar, R. Cowie, and M. Pantic, "The SEMAINE corpus of emotionally coloured character interactions," in Proc. IEEE ICME, Suntec City, Singapore, 2010, pp. 1079-1084.

[22] L. Rabiner and B. H. Juang, Fundamentals of Speech Recognition. Englewood Cliffs, NJ, USA: Prentice Hall, Apr. 1993.

[23] F. Zhou and F. De la Torre Frade, "Generalized time warping for multi-modal alignment of human motion," in Proc. IEEE CVPR, Providence, RI, USA, Jun. 2012, pp. 1282-1289.

[24] M. A. Larkin et al., "Clustal W and clustal X version 2.0," Bioinformatics, vol. 23, no. 21, pp. 2947-2948, 2007.

[25] S. Yu, K. Yu, V. Tresp, H.-P. Kriegel, and M. Wu, "Supervised probabilistic principal component analysis," in Proc. 12th ACM SIGKDD Int. Conf. KDD, New York, NY, USA, 2006, pp. 464-473.

[26] R. J. Aumann, "Agreeing to disagree," Ann. Statist., vol. 4, no. 6, pp. 1236-1239, 1976.

[27] J. Shi and J. Malik, "Normalized cuts and image segmentation," IEEE Trans. Pattern Anal. Mach. Intell., vol. 22, no. 8, pp. 888-905, Aug. 2000.

[28] I. Patras and M. Pantic, "Particle filtering with factorized likelihoods for tracking facial features," in Proc. IEEE Int. Conf. Autom. FGR, Washington, DC, USA, 2004, pp. 97-102.

[29] M. E. Tipping, "Sparse Bayesian learning and the relevance vector machine," J. Mach. Learn. Res., vol. 1, pp. 211-244, Jun. 2001.

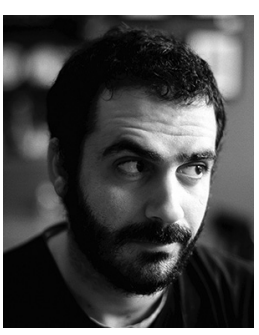

Mihalis A. Nicolaou received his B.Sc. (Ptychion) in Informatics and Telecommunications from the University of Athens, Greece in 2008 and his M.Sc. in Advanced Computing from Imperial College London, London, U.K. in 2009, both in highest honors. Mihalis is currently working towards the Ph.D. degree as a Research Assistant at Imperial College London. He has received several awards and scholarships during his studies. His current research interests lie in the areas of probabilistic component analysis and time-series analysis, with a particular focus on the application of continuous and dimensional emotion analysis.

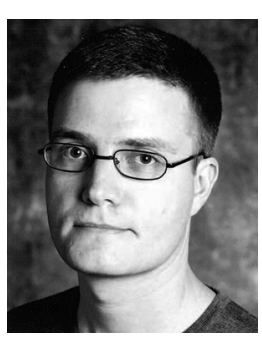

Vladimir Pavlovic is an Associate Professor in the Computer Science Department at Rutgers University, Piscataway, NJ, USA. He received the Ph.D. in electrical engineering from the University of Illinois in Urbana-Champaign in 1999. From 1999 until 2001 he was a member of research staff at the Cambridge Research Laboratory, Cambridge, MA, USA. Before joining Rutgers in 2002, he held a research professor position in the Bioinformatics Program at Boston University. Vladimir's research interests include probabilistic system modeling, time-series analysis, statistical computer vision and bioinformatics. He has published over 100 peerreviewed papers in major computer vision, machine learning and pattern recognition journals and conferences.

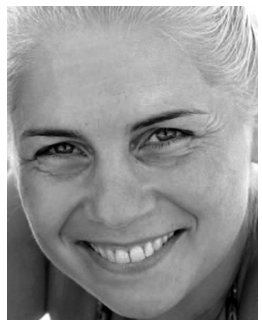

Maja Pantic is Professor in Affective and Behavioral Computing at Imperial College London, Department of Computing, London, U.K., and at the University of Twente, Department of Computer Science, The Netherlands. She received various awards for her work on automatic analysis of human behavior including the Roger Needham Award 2011. She currently serves as the Editor in Chief of Image and Vision Computing Journal and as an Associate Editor for both the IEEE Transactions on Systems, Man, and Cybernetics Part B and the IEEE Transactions on Pattern Analysis and Machine Intelligence. She is a Fellow of the IEEE.

For more information on this or any other computing topic, please visit our Digital Library at www.computer.org/publications/dlib. 